\title{
The monoaminergic footprint of depression and psychosis in dementia with Lewy bodies compared to Alzheimer's disease
}

Yannick Vermeiren ${ }^{1}$, Debby Van Dam¹, Tony Aerts ${ }^{1}$, Sebastiaan Engelborghs ${ }^{1,2}$, Jean-Jacques Martin ${ }^{4}$ and Peter P De Deyn ${ }^{1,2,3,4^{*}}$

\begin{abstract}
Introduction: Depression and psychosis are two of the most severe neuropsychiatric symptoms (NPS) in dementia with Lewy bodies (DLB) and Alzheimer's disease (AD). Both NPS have negative effects on cognitive performance and life expectancy. The current study aimed to investigate and compare monoaminergic etiologies between both neurodegenerative conditions, given the lack of an efficient pharmacological treatment until present.

Methods: Eleven behaviorally relevant brain regions of the left frozen hemisphere of 10 neuropathologically confirmed $A D$ patients with/without depression ( $A D+D /-D ; 5$ were psychotic within $A D+D), 10$ confirmed DLB patients, all of whom were depressed (DLB + D; 5 psychotic patients), and, finally, 10 confirmed control subjects were regionally dissected. All patients were retrospectively assessed before death using the Behavioral Pathology in Alzheimer's Disease Rating Scale (Behave-AD) and Cornell Scale for Depression in Dementia amongst others. The concentrations of dopamine (DA), serotonin (5-HT), (nor)adrenaline and respective metabolites, i.e. 3,4-dihydroxyphenylacetic acid (DOPAC) and homovanillic acid (HVA), 5-hydroxy-3-indoleacetic acid (5-HIAA), and, 3-methoxy-4-hydroxyphenylglycol (MHPG), were determined using reversed-phase high-performance liquid chromatography with electrochemical detection.

Results: DLB subjects had the overall lowest monoamine and metabolite concentrations regarding 33 out of 41 significant monoaminergic group alterations. Moreover, MHPG levels were significantly decreased in almost 8 out of 11 brain regions of DLB- compared to AD patients. We also observed the lowest 5-HT and 5-HIAA levels, and 5-HIAA/5-HT turnover ratios in DLB + D compared to AD + D subjects. Additionally, a 4- and 7-fold increase of DOPAC/DA and HVA/ DA turnover ratios, and, a 10-fold decrease of thalamic DA levels in DLB + D compared to AD + D patients and control subjects was noticed. Regarding psychosis, hippocampal DA levels in the overall DLB group significantly correlated with Behave-AD AB scores. In the total AD group, DA levels and HVA/DA ratios in the amygdala significantly correlated with Behave-AD AB scores instead.

Conclusions: Monoaminergic neurotransmitter alterations contribute differently to the pathophysiology of depression and psychosis in DLB as opposed to $A D$, with a severely decreased serotonergic neurotransmission as the main monoaminergic etiology of depression in DLB. Similarly, psychosis in DLB might, in part, be etiologically explained by dopaminergic alterations in the hippocampus, whereas in AD, the amygdala might be involved.
\end{abstract}

\footnotetext{
* Correspondence: dedeyn@skynet.be

'Laboratory of Neurochemistry and Behavior, Institute Born-Bunge, University of Antwerp, Campus Drie Eiken, Universiteitsplein 1, Wilrijk, Antwerp 2610, Belgium ${ }^{2}$ Department of Neurology and Memory Clinic, Hospital Network Antwerp (ZNA) Middelheim and Hoge Beuken, Lindendreef 1, Antwerp 2020, Belgium Full list of author information is available at the end of the article
}

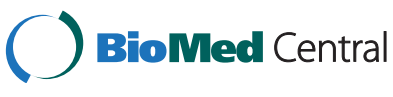

(c) 2015 Vermeiren et al.; licensee BioMed Central. This is an Open Access article distributed under the terms of the Creative Commons Attribution License (http://creativecommons.org/licenses/by/4.0), which permits unrestricted use, distribution, and reproduction in any medium, provided the original work is properly credited. The Creative Commons Public Domain Dedication waiver (http://creativecommons.org/publicdomain/zero/1.0/) applies to the data made available in this article, unless otherwise stated. 


\section{Introduction}

Dementia with Lewy bodies (DLB) is the second most common neurodegenerative disorder following Alzheimer's disease (AD) and accounts for up to $20 \%$ of all autopsy-confirmed dementias in the elderly $[1,2]$. One of the key hallmarks of DLB patients, besides the cognitive impairment and parkinsonian symptomatology, is the high frequency of neuropsychiatric symptoms (NPS), particularly psychosis [3]. The presence of recurrent visual hallucinations has even been identified as one of the core features in the clinical diagnosis of DLB. Additionally, the supportive and suggestive diagnostic features include depression, systematized delusions and rapid eye movement (REM) sleep behavior disorders among others [4]. Typically, visual hallucinations, delusions and depression are much more common in DLB than in AD [5,6]. The first two NPS occur in approximately $60 \%$ to $70 \%$ of DLB patients [5-7], whereas depression, although frequently present in $\mathrm{AD}(28 \%)$, remains much more persistent in DLB (45\%) after a follow-up period of one year [8]. Moreover, depressive symptoms in AD and DLB are associated with a greater cognitive decline [8] and, in $\mathrm{AD}$, significantly relate to lower survival rates over a three-year period [9]. Psychosis in AD is also very common and has previously been associated with an increased mortality rate and, again, an accelerated cognitive decline [10,11]. Besides depression and psychosis, symptoms of anxiety, apathy and sleep disturbances often coexist in DLB patients $[3,12]$. In addition, delusions and hallucinations may trigger other NPS, such as agitation or aggression, which regularly leads to early nursing home admission [12].

At present, regarding the different classes of psychoactive drug therapies to alleviate depression and psychosis in $\mathrm{AD}$, antipsychotics are the primary pharmacological treatment option, although they may induce serious side effects, increase mortality rates [13], and their efficacy is 'modest' at best [14]. The administration of psychotropic medication has also been associated with a more rapid cognitive and functional decline, and not necessarily with improved NPS [15]. As for antidepressants, Pollock and colleagues [16] reported that citalopram was superior to placebo, with greatest efficacy for aggression, and, in a later study [17], citalopram was found to be comparable in efficacy to risperidone, differentiated by its significant effect on agitation and its superior tolerability in the treatment of moderate to severe NPS. Randomized controlled trials of sertraline [18] and trazodone [19], however, have been less promising. The pharmacological treatment of NPS in DLB patients, on the other hand, requires an even more cautious approach. For example, all drugs with anticholinergic side effects, such as tricyclic antidepressants, low potency neuroleptics, antiparkinsonian anticholinergic drugs and antispasmodics for bladder or gastrointestinal tract, should be avoided due to their potential to exacerbate psychotic symptoms and, moreover, might induce orthostatic hypotension [12]. Consequently, cholinomimetic therapy using cholinesterase inhibitors has proven to be beneficial for apathy, anxiety and psychosis [20-22], while two other studies found weak [23] or unclear [24] evidence to support its use in DLB compared to Parkinson's disease dementia (PDD). Interestingly, concurrent treatment with a selective serotonin reuptake inhibitor (SSRI) and a serotonin (5-hydroxytryptamine, 5-HT) 1A receptor antagonist might offer a positive outcome to treat depression efficiently in DLB, as was suggested by Francis [25], although evidence for the benefits of antipsychotics other than clozapine is limited, and there are serious safety concerns about the use of antipsychotics in these patients [3]. Finally, the administration of memantine, an $\mathrm{N}$-methyl-D-aspartate (NMDA) receptor antagonist, may result in variable symptomatic side effects in DLB patients, including worsening of psychosis or even an adverse drug reaction $[26,27]$.

While the neurobiological aspects of the parkinsonism of DLB patients have been intensively studied, much less attention has been paid to the pathophysiological mechanisms underlying depression and psychosis associated with DLB. More specifically, very few studies have attempted to delineate their neurochemical correlates [25,28-31] and whether these are similar to or distinct from $\mathrm{AD}$ [25]. Furthermore, given the lack of an efficient psychotropic monoaminergic therapy, and due to the fact that psychosis and depression are enormously troublesome to caregivers and patients, cause an earlier institutionalization and account for a significant increase in the overall cost of dementia, it becomes essential to intensely investigate the monoaminergic pathophysiology of NPS features in DLB compared to AD. Likewise, although recently a handful of studies have examined the monoaminergic etiology of NPS in AD, as well as in DLB and frontotemporal dementia, in postmortem brain tissue [32] and cerebrospinal fluid (CSF) samples [33], a neurochemical comparison study between DLB and AD supplemented with the inclusion of an age- and gender-matched control group would give more insight into the monoaminergic characteristics of depression and psychosis of both neurodegenerative disorders separately. In the long term, such comparison studies may contribute to the development of novel psychotropic pharmacotherapies [3,34].

We, therefore, determined the levels of eight monoamines and metabolites, that is, the indolamine 5- $\mathrm{HT}$, the catecholamines dopamine (DA), adrenaline (A) and noradrenaline (NA), as well as their respective metabolites, that is, 5-hydroxy-3-indoleacetic acid (5-HIAA; metabolite of the serotonergic neurotransmitter system), 3,4-dihydroxyphenylacetic acid and homovanillic acid 
(DOPAC and HVA, respectively; metabolites of the dopaminergic neurotransmitter system) and 3-methoxy-4hydroxyphenylglycol (MHPG; metabolite of the (nor) adrenergic neurotransmitter system) in various postmortem brain regions of depressed and/or psychotic DLB and $\mathrm{AD}$ patients using reversed-phase high-performance liquid chromatography with electrochemical detection (RP-HPLC-ECD) to identify monoaminergic neurotransmitter alterations which may underlie both NPS. As a baseline reference, tissue samples of the same brain regions of a healthy control group were neurochemically analyzed as well.

\section{Methods}

\section{Study population and inclusion protocol}

Neuropathologically confirmed AD patients with $(n=10$; $\mathrm{AD}+\mathrm{D})$ and without $(\mathrm{n}=10 ; \mathrm{AD}-\mathrm{D})$ depression, 10 confirmed DLB patients, all of whom were depressed $(\mathrm{DLB}+\mathrm{D})$ and, finally, 10 confirmed control subjects were selected from the Biobank of the Institute Born-Bunge (University of Antwerp, Antwerp, Belgium). Initially, all patients with a clinical diagnosis of probable $\mathrm{AD}$ and DLB were recruited at the Memory Clinic of the Hospital Network Antwerp (ZNA-Middelheim and ZNA-Hoge Beuken, Antwerp, Belgium) for inclusion in a prospective, longitudinal study on NPS [35]. As part of their differential diagnostic work-up of dementia, besides the general physical and neurological examinations, blood screening tests, structural neuroimaging by computed tomography (CT), magnetic resonance imaging (MRI) or single photon emission computed tomography (SPECT), neuropsychological evaluations (for example, Mini-Mental State Examination (MMSE) scores) and optional cerebrospinal fluid (CSF)/blood sampling for biomarker and/or DNA analyses, a baseline behavioral assessment was routinely performed. If possible, AD and DLB patients were behaviorally rated again during follow-up. The clinical diagnosis of probable $\mathrm{AD}$ was based on the NINCDS/ADRDA criteria of McKhann et al. [36,37] whereas probable DLB was diagnosed according to the consensus guidelines of McKeith et al. [4,38]. All patients also fulfilled the Diagnostic and Statistical Manual of Mental Disorders - IV text revision (DMS-IV-TR) criteria [39]. On the other hand, age-matched control subjects were hospitalized in the Middelheim General Hospital (Antwerp, Belgium) and consented shortly before death. Death causes were cardiac failure due to an acute myocardial infarct $(n=2)$, chronic obstructive pulmonary disease (COPD) $(\mathrm{n}=3)$, carcinoma (hepatic $(\mathrm{n}=1)$; prostate $(\mathrm{n}=1)$; lung $(\mathrm{n}=1)$; neuroendocrine $(\mathrm{n}=1))$ and multiple myeloma $(\mathrm{n}=1)$. Moreover, clinical neurological examination, as well as a retrospective review of the clinical history, neuropsychological evaluation and hospital records, did not reveal any evidence of dementia, psychiatric antecedents or cognitive decline. Written informed consent regarding autopsy and the subsequent use of brain tissue, clinical documentation and behavioral data for research purposes was obtained from all participants. The study was also approved by the Medical Ethical Committee of the Middelheim General Hospital (Antwerp, Belgium) and conducted in compliance with the Helsinki Declaration.

In case consented $\mathrm{AD}$, DLB or control subjects died, brain autopsy was performed within six hours after death after which the left hemisphere was frozen at $-80^{\circ} \mathrm{C}$ for neurochemical analyses, and the right hemisphere was formaldehyde-fixated for neuropathological examination. The $10 \mathrm{AD}+\mathrm{D}$ and $10 \mathrm{AD}-\mathrm{D}$ patients were part of a larger cohort of 40 behaviorally characterized AD patients who were previously subjected to RP-HPLC-ECD assessments [32]. However, the inclusion of DLB patients and control subjects, as well as the inclusion of several extra brain regions, render the current study significantly distinct from our previous one [32], which had the same methodological setup, but an entirely different hypothesis.

None of the included control subjects suffered from central nervous system pathology.

\section{Behavioral assessment}

Behavior of $\mathrm{AD}$ and DLB patients was assessed together with relatives and/or nursing staff using a battery of behavioral assessment scales, including: the Behavioral Pathology in Alzheimer's Disease Rating Scale (Behave-AD) [40]; Middelheim Frontality Score (MFS) [41]; CohenMansfield Agitation Inventory (CMAI) [42]; and Cornell Scale for Depression in Dementia (CSDD) [43]. Dementia staging was based on the Global Deterioration Scale (GDS) with a range varying from 1 (nondemented) to 7 (terminal stage of dementia) [44]. During each NPS assessment, only the behavioral phenomena covering the last two weeks prior to the assessment were included and rated. Behavioral assessment was repeated during each neurological follow-up examination in the hospital, if possible $(n=2$ for $A D+D, n=3$ for $A D-D$ and $n=6$ for $D L B+D)$. $A$ final retrospective behavioral scoring was performed in case patients died more than ten days after the last followup or baseline rating. In total, eight $A D+D$-, seven $A D-$ $\mathrm{D}$ - and four $\mathrm{DLB}+\mathrm{D}$ patients underwent only one rating close to death, given the short amount of time which was left since they entered our study protocol. Nevertheless, for this research purpose, only the final behavioral assessment scores around the date of death were used so that possible neurochemical alterations in the brain were as representative as possible for the concurrent clinical manifestation of NPS in all dementia patients.

Patients with a total CSDD cutoff score $\geq 8$ were classified as depressed, whereas patients with a CSDD score $<8$ were defined as non-depressed [45]. Furthermore, patients with a Behave-AD cluster A score (delusions) $\geq 4$ or a 
Behave-AD cluster B score (hallucinations) $\geq 2$ or patients who were rated on the Behave-AD cluster $\mathrm{A}$ and $\mathrm{B}$ subscales (delusions + hallucinations), irrespective of its combined value, were classified as psychotic [46]. Finally, based on their behavioral profiles, AD and DLB patients were divided into three main groups, that is, $A D+D$ $(\mathrm{n}=10$, of which 5 were psychotic $(A D+D+P), A D-D$ $(\mathrm{n}=10$, of which none were psychotic $(A D-D-P))$ and $\mathrm{DLB}+\mathrm{D}(\mathrm{n}=10$, of which 5 were psychotic $(\mathrm{DLB}+\mathrm{D}+\mathrm{P})$.

No behavioral scores were available for the control group.

\section{Neuropathological examination and criteria}

In order to neuropathologically confirm or reject the clinically established AD and DLB diagnoses, immunohistochemistry on a standard selection of 10 to 13 regionally dissected postmortem brain regions of the formaldehydefixated right hemisphere was performed [47]. In short, the stains routinely applied in our laboratory on paraffin blocks of the neocortex (frontal, temporal and occipital), amygdala, rhinencephalon (at the level of the posterior part of the amygdala and the lateral geniculate body (for hippocampus)), basal ganglia, thalamus, brain stem, substantia nigra (SN), pons at the level of the locus coeruleus (LC) and cerebellum are hematoxylin-eosin (HE), cresyl violet and Bodian's technique. The routinely applied immunostains are 4G8 (amyloid), AT8 (P-tau $181 \mathrm{P})$ and an anti-ubiquitin antibody (ubiquitin). When the presence of Lewy bodies (LB) is suspected on HE staining and ubiquitin immunoreactivity, an additional anti- $\alpha$-synuclein staining is applied.

Neuropathological assessments were established by the same neuropathologist (JJM), employing the criteria of Braak and Braak [48], Braak et al. [49] and Jellinger and Bancher [50] to decide on definite AD. The 'ABC scoring' method of Montine et al. [51] was applied to all AD brains collected after May $2011(n=5)$. Similarly, the consensus guidelines of McKeith et al. [4,38] were used for the neuropathological diagnosis of definite DLB. In case of significant concurrent AD pathology $(n=8$ out of 10 DLB patients), the likelihood that the pathological findings were associated with a DLB clinical syndrome was assessed as recommended by McKeith et al. [4], resulting in a final decision of definite DLB with limbic translational $(n=4$; including $n=2$ without AD pathology) and diffuse neocortical $(n=6)$ Lewy body subtype pathologies.

Regarding the control subjects, microscopic examination failed to detect significant degenerative changes in any of the control brains, except for a limited cerebral amyloid angiopathy, if present at all, and the sporadic presence of some neurofibrillary (pre)tangles or diffuse senile plaques, all of which were considered age-related.

\section{Regional brain dissection}

Regional brain dissection of the left frozen hemisphere was performed according to a standard procedure during which 21 brain regions were routinely dissected. With regard to this specific study design, a total of 11 behaviorally and neurochemically relevant brain areas were ultimately analyzed using RP-HPLC-ECD, that is, Brodmann area (BA) 9 and BA10 (prefrontal cortex), BA11 (orbitofrontal cortex), BA17 (occipital cortex), BA22 (temporal cortex), BA24 (anterior cingulate gyrus), amygdala, hippocampus, thalamus, cerebellar cortex and LC. All of these brain regions have previously been the subject of other NPSrelated neurochemical research in depressed and/or psychotic AD and DLB patients [25,28-31,52-56] and, moreover, are also part of integrated behavior-modifying brain circuits, such as the dopaminergic mesolimbic system among others. In addition, this careful selection comprises regions with clear-cut AD and DLB neuropathological and -imaging hallmarks (for example, BA22, hippocampus, prefrontal areas, thalamus and LC), as well as brain regions in which these lesions are less present to absent (BA17 and cerebellar cortex), but might still hold significant neurochemical alterations.

Dissections were performed on a plastic cutting board above a mixture of regular and dry ice by means of scalpel, tweezers and a Dremel ${ }^{\circledR} 200$ series rotary instrument tool according to a standard procedure. During the first part of this protocol, 15 of the 21 brain regions were meticulously dissected as they were easily accessible, that is, BA6/7/8/9/10/11/12/17/22/24/46, SN, cerebellar cortex, medulla oblongata and LC. Afterwards, the remaining left hemisphere was placed at $-20^{\circ} \mathrm{C}$ for four hours after which seven to eight coronal slices were cut. Subsequently, the remaining six brain regions were dissected, namely amygdala, hippocampus, thalamus, caudate nucleus, putamen and globus pallidus. All dissected brain tissue samples roughly weighed 300 to $500 \mathrm{mg}$ and were immediately stored on dry ice in precooled aluminum cryovials (Sanbio BV, Uden, The Netherlands) during the dissection. In total, each dissected brain region could be subdivided into four of these cryovials of which three were used for HPLC analyses. The fourth one was used for $\mathrm{pH}$ measurement if necessary.

All AD, DLB and control brain hemispheres were dissected by the same researcher to minimize variability.

\section{Neurochemical RP-HPLC-ECD analysis}

A recently optimized and validated RP-HPLC system with electrochemical detection (ECD) for the fast detection of monoaminergic compounds in human brain tissue was used to simultaneously measure the concentrations of $5-\mathrm{HT}, \mathrm{DA},(\mathrm{N}) \mathrm{A}$ and their respective metabolites, that is, 5-HIAA, DOPAC/HVA and MHPG [57]. Sample analysis was performed using an Alexys ${ }^{\mathrm{TM}}$ Dual 
Monoamines Analyzer (Antec Leyden BV, Zoeterwoude, The Netherlands) by which each brain tissue sample was directly analyzed in duplicate. Output ranges were 500 $\mathrm{pA}$ and $2 \mathrm{nA}$, respectively, with two electrochemical VT03 flow cells each containing a glassy carbon working electrode of $0.7 \mathrm{~mm}$ and an in situ $\mathrm{Ag} / \mathrm{AgCl}$ reference electrode at $670 \mathrm{mV}$ placed in a Decade II electrochemical detector (Antec Leyden BV, Zoeterwoude, The Netherlands). An isocratic flow rate of $40 \mu \mathrm{L}$ of mobile phase per minute was set for both LC 110 pumps. The optimal conditions for separation of the monoaminergic compounds were obtained using a mobile phase comprising $13 \%$ methanol combined with a mixture of phosphoric $(50 \mathrm{mM})$ and citric acid $(50 \mathrm{mM})$, octane-1-sulfonic acid sodium salt $(1.8 \mathrm{mM}), \mathrm{KCl}(8 \mathrm{mM})$ and ethylenediaminetetraacetic acid (EDTA) (0.1 mM) (pH 3.6). Samples were loaded onto two microbore ALF-125 columns $(250 \mathrm{~mm} \times$ $1.0 \mathrm{~mm}, 3 \mu \mathrm{m}$ particle size) filled with a porous C18 silica stationary phase by an Alexys ${ }^{\mathrm{TM}}$ AS 100 Autosampler (Antec Leyden BV, Zoeterwoude, The Netherlands). Separation of the monoamines and metabolites was achieved at a stable column and VT03 flow cell temperature of $36^{\circ} \mathrm{C}$ with a total runtime of only 45 minutes per sample. Levels of the monoaminergic compounds were calculated using Clarity ${ }^{\mathrm{TM}}$ Software (DataApex Ltd, 2008, Prague, The Czech Republic). All chemicals were of analytical grade.

The brain sample preparation procedure prior to RPHPLC-ECD analysis was simple and fast. Initially, 200 to $300 \mathrm{mg}$ frozen brain tissue was weighed in $4 \mathrm{ml}$ mobile phase. Next, this mixture was homogenized for 40 seconds (50 seconds in case samples that weighed $\geq 250 \mathrm{mg}$ ) at moderate speed using an Ultra-Turrax TR 50 homogenizer ${ }^{\circledR}$ (Janke \& Kunkel, Ika-Werk, Staufen, Germany). The homogenate was then centrifuged $\left(26,000 \times \mathrm{g}, 20\right.$ minutes, $\left.4^{\circ} \mathrm{C}\right)$ and afterwards, the supernatant was filtered using a $0.2 \mu \mathrm{m}$ Millipore $^{\bullet}$ filter (Millex, Merck Millipore, Carrigtwohill, County Cork, Ireland) attached to a syringe. Further elimination of proteins was accomplished using $10 \mathrm{kDa}$ Amicon $^{\odot}$ Ultra Centrifugal Filters (Millipore) $(14,000 \times \mathrm{g}$, 20 minutes, $4^{\circ} \mathrm{C}$ ) which were washed twice beforehand with mobile phase. The final filtrate was then loaded onto the HPLC columns (undiluted and diluted).

\section{$\mathrm{pH}$ measurement of cerebellar brain tissue}

Samples needed to be nonacidotic (that is, $\mathrm{pH}>6.1$ ) $[58,59]$ in order to guarantee high-quality brain tissue since acidosis may induce alterations in neurotransmitter and neuropeptide concentrations, as well as enzyme activity. Several factors such as a prolonged death struggle, premortem stroke and a long postmortem delay could acidify brain tissue $[60,61]$. In this study, pH values of the cerebellar cortex were measured since the cerebellar $\mathrm{pH}$ has previously been shown to be most representative for the entire brain [61].
First, a $0.01 \mathrm{~N} \mathrm{NaOH}$-solution was prepared to adjust the $\mathrm{pH}$ of deionized water to 7.0. Next, approximately $100 \mathrm{mg}$ of frozen cerebellar cortex was weighed after which $1 \mathrm{ml}$ of the adjusted water was added. The mixture was then homogenized with a Pro-200 Homogenizer (Pro Scientific, Oxford, CT, USA) for 30 seconds at moderate speed at $4^{\circ} \mathrm{C}$. Finally, the homogenate was centrifuged $\left(8,000 \times \mathrm{g}, 10\right.$ minutes, $\left.4^{\circ} \mathrm{C}\right)$ and the $\mathrm{pH}$ of the supernatant was measured. Per patient, two cerebellar samples were included.

At the end, all brain regions with acidotic $\mathrm{pH}$ values $(<6.1)$ were excluded from data analysis.

\section{Statistical analysis}

Nonparametric statistics were applied due to the limited number of patients, ordinal variables (behavioral scores) and not normally distributed data after performing a Shapiro-Wilk test of normality.

Chi-square statistics were applied to compare male/ female ratios and patients taking/not taking psychotropic medication across groups. Kruskal-Wallis analyses with post-hoc Mann-Whitney U tests were used for comparison of all behavioral, neurocognitive, demographic, $\mathrm{pH}$ and monoaminergic data between $\mathrm{AD}+\mathrm{D}, \mathrm{AD}-\mathrm{D}, \mathrm{DLB}+\mathrm{D}$ and control subjects, as well as the monoaminergic data between $\mathrm{AD}+\mathrm{D}+\mathrm{P}, \mathrm{AD}+\mathrm{D}-\mathrm{P}, \mathrm{DLB}+\mathrm{D}+\mathrm{P}$ and $\mathrm{DLB}+$ $\mathrm{D}-\mathrm{P}$. In all cases, only data remaining statistically significant following a Bonferroni correction for multiple comparisons $(P<0.017$ for three group comparisons (Table 1 ) and $P<0.0083$ for six group comparisons (Tables 2 and 3)) were regarded as significant. Mann-Whitney U tests were used to look at potential confounding effects of psychotropic medication, with regard to our neurochemical data, between patients taking and not taking psychotropic medication within each group. Finally, in order to calculate neurochemical correlations of CSDD-, Behave-AD cluster $\mathrm{A} / \mathrm{B} / \mathrm{A}+\mathrm{B}$ - and other behavioral scores in the total AD $(\mathrm{n}=20)$ and DLB $(\mathrm{n}=10)$ populations, nonparametric Spearman's Rank-Order correlation statistics were applied. Again, a Bonferroni correction was performed, and only those significant data were taken into account (Table 4; $P<0.0033)$. All analyses were performed using SPSS 22.0 for Windows (IBM SPSS Software, Armonk, NY, USA, IBM Corp.). Figures were generated using GraphPad Prism 6.0 software.

\section{Results}

Clinical and MMSE data, pH values and dementia staging

Table 1 summarizes the clinical, MMSE, $\mathrm{pH}$ and GDS data.

All groups were age- and gender-matched, with similar storage times of the analyzed brain tissue samples and postmortem delays. The number of patients who were on psychotropic medication before death compared to the patients who were free of such medication was 
Table 1 Clinical data, behavioral assessment scores and $\mathrm{pH}$ values

\begin{tabular}{|c|c|c|c|c|c|}
\hline Parameter & $\begin{array}{l}A D+D \\
(\text { number }=10)\end{array}$ & $\begin{array}{l}\text { AD-D } \\
\text { (number = 10) }\end{array}$ & $\begin{array}{l}\text { DLB + D } \\
\text { (number = 10) }\end{array}$ & $\begin{array}{l}\text { CONTR } \\
\text { (number = 10) }\end{array}$ & Kruskal Wallis \\
\hline \multicolumn{6}{|l|}{ Demographics and clinical data } \\
\hline Age at onset dementia (years) & $71.5 \pm 14.0(53-94)$ & $79.4 \pm 13.5(50-96)$ & $76.0 \pm 5.8(66-84)$ & N/A & $P=0.286$ \\
\hline Age at death (years) & $75.7 \pm 12.3(58-95)$ & $83.5 \pm 11.6(59-97)$ & $81.4 \pm 4.4(72-87)$ & $76.5 \pm 7.0(65-87)$ & $P=0.148$ \\
\hline Gender, male/female (number) & $6 / 4$ & $7 / 3$ & $7 / 3$ & $5 / 5$ & $\begin{aligned} X^{2} & =1.173 \\
P & =0.759\end{aligned}$ \\
\hline Storage time tissue at $-80^{\circ} \mathrm{C}$ (years) & $4.3 \pm 3.8(0.3-10)$ & $4.1 \pm 4.0(0.6-10)$ & $6.6 \pm 4.3(2-11)$ & $8.6 \pm 1.0(7-10)$ & $P=0.060$ \\
\hline Postmortem delay ${ }^{\complement}$ (hours) & $3.7 \pm 1.0(2-5)$ & $3.7 \pm 0.9(2-6)$ & $4.2 \pm 1.4(2-6)$ & $4.8 \pm 1.6(3-6)$ & $P=0.384$ \\
\hline $\begin{array}{l}\text { Taking/not taking psychotropic } \\
\text { medication (number) }\end{array}$ & $5 / 5$ & $7 / 3$ & $6 / 4$ & $4 / 6$ & $\begin{array}{l}X^{2}=1.125 \\
P=0.771\end{array}$ \\
\hline \multicolumn{6}{|l|}{ Behavioral assessment scores } \\
\hline MFS total score $(/ 10)$ & $4.8 \pm 0.9(3-6)^{\mathrm{aa}}$ & $2.3 \pm 1.8(1-5)^{\mathrm{aa}}$ & $4.3 \pm 1.3(3-7)$ & N/A & $P=0.008$ \\
\hline Behave-AD cluster A (/21) & $1.4 \pm 2.4(0-6)$ & $0.10 \pm 0.3(0-1)$ & $0.4 \pm 0.8(0-2)$ & N/A & $P=0.401$ \\
\hline \multicolumn{6}{|l|}{ Paranoid and delusional ideation } \\
\hline Behave-AD cluster B (/15) & $0.8 \pm 1.2(0-3)$ & $0.0 \pm 0.0^{b}$ & $1.9 \pm 2.2(0-6)^{b}$ & N/A & $P=0.033$ \\
\hline \multicolumn{6}{|l|}{ hallucinations } \\
\hline Behave-AD cluster AB (/36) & $2.2 \pm 2.5(0-6)$ & $0.1 \pm 0.3(0-1)^{\mathrm{b}}$ & $2.3 \pm 2.3(0-6)^{b}$ & N/A & $P=0.041$ \\
\hline \multicolumn{6}{|l|}{ psychosis } \\
\hline Behave-AD cluster C score (/9) & $2.9 \pm 2.8(0-7)$ & $0.5 \pm 0.9(0-2)$ & $0.9 \pm 1.3(0-4)$ & N/A & $P=0.084$ \\
\hline \multicolumn{6}{|l|}{ activity disturbances } \\
\hline Behave-AD cluster D score (/9) & $4.5 \pm 2.9(0-9)$ & $1.7 \pm 1.9(0-4)$ & $1.8 \pm 2.4(0-7)$ & N/A & $P=0.052$ \\
\hline \multicolumn{6}{|l|}{ aggressiveness } \\
\hline Behave-AD cluster E score (/3) & $0.7 \pm 0.8(0-2)$ & $0.1 \pm 0.3(0-1)$ & $0.3 \pm 0.7(0-2)$ & N/A & $P=0.111$ \\
\hline \multicolumn{6}{|l|}{ diurnal rhythm disturbances } \\
\hline Behave-AD cluster F score (/6) & $1.9 \pm 1.7(0-4)$ & $0.3 \pm 0.7(0-2)$ & $1.1 \pm 1.4(0-4)$ & N/A & $P=0.052$ \\
\hline \multicolumn{6}{|l|}{ affective disturbances } \\
\hline Behave-AD cluster G score (/12) & $1.2 \pm 2.0(0-5)$ & $0.0 \pm 0.0$ & $0.8 \pm 1.7(0-4)$ & N/A & $P=0.200$ \\
\hline \multicolumn{6}{|l|}{ anxieties and phobias } \\
\hline Behave-AD total score (/75) & $13.4 \pm 8.9(2-24)^{\mathrm{aa}}$ & $2.7 \pm 2.4(0-6)^{a a}$ & $7.3 \pm 5.3(2-17)$ & N/A & $P=0.009$ \\
\hline Behave-AD global score (/3) & $1.7 \pm 0.9(0-3)^{a a}$ & $0.4 \pm 0.5(0-1)^{a a}$ & $1.2 \pm 0.8(0-2)$ & $\mathrm{N} / \mathrm{A}$ & $P=0.006$ \\
\hline \multicolumn{6}{|l|}{ caregiver burden } \\
\hline CMAl cluster 1 (/70) & $15.4 \pm 8.1(10-35)$ & $10.8 \pm 2.5(10-18)$ & $11.1 \pm 2.4(10-17)$ & N/A & $P=0.041$ \\
\hline \multicolumn{6}{|l|}{ aggressive behavior } \\
\hline CMAI cluster 2 (/77) & $24.7 \pm 10.9(11-39)^{a}$ & $13.1 \pm 3.3(11-21)^{a}$ & $18.5 \pm 7.5(11-33)$ & N/A & $P=0.021$ \\
\hline \multicolumn{6}{|l|}{ physically nonaggressive behavior } \\
\hline CMAl cluster 3 (/56) & $16.1 \pm 8.6(8-33)$ & $11.8 \pm 5.6(8-23)$ & $14.9 \pm 7.6(8-25)$ & N/A & $P=0.344$ \\
\hline \multicolumn{6}{|l|}{ verbally agitated behavior } \\
\hline CMAI total sore (/203) & $56.2 \pm 22.1(29-90)$ & $35.7 \pm 7.4(29-47)$ & $44.5 \pm 12.3(29-65)$ & N/A & $P=0.040$ \\
\hline CSDD total score (/38) & $12.1 \pm 3.3(8-19)$ ааа & $5.0 \pm 1.2(3-7)^{a a a, ~ b b b ~}$ & $10.1 \pm 2.1(8-14)^{\mathrm{bbb}}$ & N/A & $P<0.0001$ \\
\hline \multicolumn{6}{|l|}{ depression } \\
\hline Interval between rating and death (days) & $3.8 \pm 10.1(0-6)$ & $3.9 \pm 7.0(0-9)$ & $5.2 \pm 9.4(0-10)$ & N/A & $P=0.713$ \\
\hline \multicolumn{6}{|l|}{ MMSE, staging and $\mathrm{pH}$ data } \\
\hline MMSE scored $(/ 30)$ & $\begin{array}{l}12.0 \pm 6.6(7-21) \\
\text { number }=4\end{array}$ & $\begin{array}{c}16.0 \pm 4.3(10-25)^{b} \\
\text { number }=8\end{array}$ & $\begin{array}{c}8.7 \pm 4.8(4-16)^{b} \\
\text { number }=6\end{array}$ & N/A & $P=0.041$ \\
\hline
\end{tabular}


Table 1 Clinical data, behavioral assessment scores and pH values (Continued)

\begin{tabular}{lcccc}
\hline GDS score: dementia staging (/7) & $6.2 \pm 0.8(5-7)$ & $5.8 \pm 1.0(4-7)$ & $6.6 \pm 0.5(6-7)$ & N/A \\
pH values cerebellar brain tissue & $6.5 \pm 0.3(5.9-7.1)$ & $6.5 \pm 0.3(6.0-6.8)$ & $6.7 \pm 0.3(6.4-7.2) \quad 6.4 \pm 0.2(6.0-6.7)$ & $P=0.300$
\end{tabular}

Mean \pm SD with minimum-maximum ranges between parentheses; Kruskal-Wallis analyses $(P<0.05)$ with post-hoc Mann-Whitney $\mathrm{U}$ tests were performed; data remaining statistically significant following Bonferroni correction are presented above with one superscript letter $(P<0.017$; for three groups comparisons because CONTR data are absent); $x^{2}$ statistics were used to compare male/female ratios and taking/not taking any type of psychotropic medication; significant differences with $P<0.01$ and $P<0.0001$ are respectively indicated with two and three repeated superscript letters; the following letters are used: ${ }^{\mathrm{a}} A D+D$ versus. $A D-D,{ }^{b} A D-D$ versus. $D L B+D$. ${ }^{\mathrm{C}}$ Postmortem delay indicates the number of hours between time of death and storage of the brain at $-80^{\circ} \mathrm{C}$; ${ }^{\mathrm{d}}$ only $\mathrm{MMSE}$ scores with no more than four months between scoring and date of death were included.

$A D+D /-D$, depressed/nondepressed Alzheimer's disease patients; Behave-AD, Behavioral Pathology in Alzheimer's Disease Rating Scale; CMAI, Cohen-Mansfield Agitation Inventory; CONTR; control subjects; CSDD, Cornell Score for Depression in Dementia; DLB + D, depressed dementia with Lewy bodies patients; GDS, Global Deterioration Scale; MFS, Middelheim Frontality Score; MMSE, Mini-Mental State Examination; N/A, not applicable.

comparable between groups as well. The different types of administered psychotropic medication were cholinomimetics $(\mathrm{n}=1$ for $\mathrm{AD}+\mathrm{D} ; \mathrm{n}=2$ for $\mathrm{AD}-\mathrm{D} ; \mathrm{n}=3$ for $\mathrm{DLB}+\mathrm{D})$, prolopa/levodopa $(\mathrm{n}=3$ for $\mathrm{DLB}+\mathrm{D})$, antidepressants $(n=4$ for $A D+D ; n=1$ for $A D-D ; n=3$ for $\mathrm{DLB}+\mathrm{D} ; \mathrm{n}=2$ for controls), hypnotics/sedatives/anxiolytics $(\mathrm{n}=1$ for $\mathrm{AD}+\mathrm{D}$ and $\mathrm{DLB}+\mathrm{D} ; \mathrm{n}=2$ for $\mathrm{AD}-\mathrm{D}$; $\mathrm{n}=3$ for controls) and antipsychotics $(\mathrm{n}=4$ for $\mathrm{AD}+\mathrm{D}$ and $A D-D ; n=2$ for DLB $+D)$. The average number of days between the last behavioral assessment and date of death was 3.8, 3.9 and 5.2 days for the $A D+D, A D-D$ and $\mathrm{DLB}+\mathrm{D}$ groups, respectively. Furthermore, MMSE scores of the DLB +D group were significantly lower than those of the AD-D group $(P=0.013)$, who had the overall highest MMSE scores. Finally, GDS scores and $\mathrm{pH}$ values were comparable between groups with, in total, one $\mathrm{AD}+\mathrm{D}$ and one $\mathrm{AD}-\mathrm{D}$ patient who had low cerebellar $\mathrm{pH}$ values $(<6.1)$. For these patients, extra $\mathrm{pH}$ analyses on the ten remaining brain regions were performed. Eventually, brain samples with acidotic $\mathrm{pH}$ values were excluded from statistical analysis, that is, for BA9 $(\mathrm{n}=1)$, BA11 $(\mathrm{n}=1)$, BA17 $(\mathrm{n}=1)$, amygdala $(\mathrm{n}=1)$ and cerebellum $(n=2)$.

\section{Behavioral assessment scores}

The behavioral assessment scores of each group are presented in Table 1.

The MFS total scores $(P=0.004)$, Behave-AD total scores $(P=0.005)$, Behave-AD global scores $(P=0.004)$, CMAI cluster 2 scores $(P=0.015)$ and CSDD total scores $(P=0.00001)$ were significantly higher in the $\mathrm{AD}+\mathrm{D}$ group compared to the AD-D group, whereas the BehaveAD cluster $\mathrm{B}$ scores $(P=0.013)$, Behave-AD cluster $\mathrm{AB}$ scores $(P=0.013)$ and CSDD total scores $(P=0.00001)$ were all significantly lower in the AD-D group compared to the DLB + D group.

Focus depression: neurochemical comparison of brain monoamines, metabolites and ratios between $A D+D$, $A D-D, D L B+D$ and control subjects

Only the neurochemical group differences which remained statistically significant following Bonferroni correction are displayed in Table $2(P<0.00833)$. Nonsignificant data were omitted.

The DLB + D group had the overall lowest monoamine and metabolite levels, as well as ratios. More specifically, the DLB + D group had the significantly lowest values for 33 out of 41 monoaminergic group alterations (rows), across 11 brain regions, compared to $\mathrm{AD}+\mathrm{D}$, AD-D and/or control subjects (Table 2). In addition, the $\mathrm{DLB}+\mathrm{D}$ group had the significantly highest DOPAC/DA and HVA/DA ratios, indicative of dopaminergic turnover, specifically for hippocampus (only DOPAC/DA), thalamus (both), and cerebellum (only DOPAC/DA), as well as the highest MHPG/NA ratios, indicative of noradrenergic catabolism, in the LC. The control group had the lowest, second lowest and highest values for 5, 18 and 7 out of 41 significant monoaminergic group differences, respectively. As for the $\mathrm{AD}+\mathrm{D} /$ - $\mathrm{D}$ groups, levels varied, but, in general, they had the highest concentrations for 29 rows out of the total of 41 .

The most statistically significant alterations were noticeable in the thalamus, with significantly lower DA levels and, at the same time, significantly increased DOPAC/DA and HVA/DA ratios in the DLB $+\mathrm{D}$ population compared to the $\mathrm{AD}+\mathrm{D}(P=0.00008,0.002$ and 0.007, respectively), AD-D $(P=0.0003,0.002$ and 0.007, respectively) and control group $(P=0.00008,0.0003$ and 0.004, respectively). Thalamic NA levels were also significantly decreased $(P=0.003,0.001$ and 0.0005 , respectively), whereas thalamic MHPG levels of the DLB +D group were only significantly lower when compared to the $\mathrm{AD}+\mathrm{D}$ population $(P=0.0002)$. Similarly, 5 -HT levels of BA9, BA10 and BA24, as well as 5-HIAA levels of BA10, BA17, BA22 and BA24 were significantly lower in DLB + $\mathrm{D}$ patients compared to their AD + D (for 5-HT: $P=0.001$, 0.00001 and 0.0005 ; for 5-HIAA: $P=0.003,0.00008$, 0.0004 and 0.007 , respectively) and AD-D (for 5-HT: $P=0.00008,0.00008$ and 0.0003 ; for 5-HIAA: $P=0.029$, $0.002,0.001$ and 0.007 , respectively) counterparts. The hippocampal 5-HIAA/5-HT ratios, indicative of serotonergic catabolism, were significantly decreased in $\mathrm{DLB}+\mathrm{D}$ patients compared to $\mathrm{AD}+\mathrm{D}$ patients as well $(P=0.003)$. Furthermore, in the LC, DOPAC, DA and HVA levels of the $\mathrm{DLB}+\mathrm{D}$ population were significantly decreased 
Table 2 Focus depression: comparison of the brain monoamine levels between $A D+D, A D-D, D L B+D$ and CONTR

\begin{tabular}{|c|c|c|c|c|c|}
\hline Brain region & MA and MT or ratio & $A D+D($ number $=10)$ & $A D-D$ (number = 10) & $\mathrm{DLB}+\mathrm{D}($ number $=10)$ & CONTR (number $=10$ ) \\
\hline \multirow[t]{2}{*}{ BA9 } & MHPG (ng/g) & $\begin{array}{c}412.4(314.3-704.8) \\
n=9\end{array}$ & $\begin{array}{c}\text { 1,092.3 (499.2-1,734.5); } \\
n=10^{\mathrm{b}}\end{array}$ & $\begin{array}{c}284.3(155.8-547.0) \\
n=10^{b}\end{array}$ & $\begin{array}{c}478.7(409.2-758.3) \\
n=10\end{array}$ \\
\hline & $5-\mathrm{HT}(\mathrm{ng} / \mathrm{g})$ & $\begin{array}{c}9.8(5.3-16.9) \\
n=9^{a}\end{array}$ & $\begin{array}{l}12.6(7.7-14.4) \\
n=10^{\mathrm{e}}, \mathrm{bbb}\end{array}$ & $\begin{array}{l}3.4(2.5-4.2) \\
\mathrm{n}=10^{\mathrm{a}}, \mathrm{bbb}\end{array}$ & $\begin{array}{c}4.2(1.3-11.0) \\
n=10^{\mathrm{e}}\end{array}$ \\
\hline \multirow[t]{4}{*}{ BA10 } & MHPG (ng/g) & $\begin{array}{c}684.2(312.1-1,080.1) \\
n=10\end{array}$ & $\begin{array}{c}663.9(432.5-940.3) \\
n=10^{b}\end{array}$ & $\begin{array}{c}297.7\left(\begin{array}{c}(143.5-460.4) \\
n=10^{b}\end{array}\right.\end{array}$ & $\begin{array}{c}289.9(177.3-498.1) \\
n=10\end{array}$ \\
\hline & 5-HIAA (ng/g) & $\begin{array}{c}239.2(156.9-367.3) \\
n=10^{\mathrm{a}}\end{array}$ & $\begin{array}{c}178.5(124.3-310.5) \\
n=10\end{array}$ & $\begin{array}{l}82.1(58.9-125.8) ; \\
n=10^{\mathrm{a}}\end{array}$ & $\begin{array}{c}128.7(85.8-157.0) ; \\
n=10\end{array}$ \\
\hline & HVA (ng/g) & $\begin{array}{c}116.6(89.2-132.0) \\
n=10^{\mathrm{a}}\end{array}$ & $\begin{array}{c}82.8(63.2-98.0) \\
\quad n=10\end{array}$ & $\begin{array}{c}57.3(41.9-66.3) \\
n=10^{\mathrm{a}}\end{array}$ & $\begin{array}{c}102.6(66.1-138.7) ; \\
n=10\end{array}$ \\
\hline & 5-HT (ng/g) & $\begin{array}{c}16.0(11.8-34.4) \\
n=10^{\text {aaa }}\end{array}$ & $\begin{array}{c}13.4(12.7-15.4) \\
n=10^{\mathrm{bbb}}\end{array}$ & $\begin{array}{c}4.3(2.4-5.8) ; \\
n=10^{\text {aaa,bbb, } f}\end{array}$ & $\begin{array}{c}12.4(8.6-18.3) \\
n=10^{f}\end{array}$ \\
\hline \multirow[t]{3}{*}{ BA24 } & 5-HIAA (ng/g) & $\begin{array}{c}394.5(300.9-565.8) \\
n=10^{\mathrm{a}}\end{array}$ & $\begin{array}{c}378.2(360.6-508.6) \\
n=10^{b}\end{array}$ & $\begin{array}{c}229.5(194.6-335.2) \\
n=10^{a, b}\end{array}$ & $\begin{array}{c}301.2(192.1-499.8) \\
n=10\end{array}$ \\
\hline & HVA (ng/g) & $\begin{array}{c}214.5(180.5-263.2) \\
n=10^{\mathrm{a}}\end{array}$ & $\begin{array}{c}202.1(144.0-224.2) \\
n=10\end{array}$ & $\begin{array}{c}131.21(100.5-183.1) \\
n=10^{\mathrm{a}}\end{array}$ & $\begin{array}{c}210.6(179.8-263.6) \\
n=10\end{array}$ \\
\hline & 5-HT (ng/g) & $\begin{array}{c}39.4(32.0-54.1) \\
n=10^{\text {aa }}\end{array}$ & $\begin{array}{c}44.4(34.7-52.9) \\
n=10^{\mathrm{bb}}\end{array}$ & $\begin{array}{c}21.6(8.4-23.5) \\
n=10^{\text {aa, bb }}\end{array}$ & $\begin{array}{c}42.6(22.0-53.0) \\
n=10\end{array}$ \\
\hline \multirow[t]{2}{*}{ amygdala } & MHPG (ng/g) & $\begin{array}{c}1,100.5(634.4-1,568.7) \\
n=9^{a}\end{array}$ & $\begin{array}{c}592.2(209.1-1,050.7) \\
n=8\end{array}$ & $\begin{array}{c}430.1 \begin{array}{c}(137.3-644.2) \\
n=10^{\mathrm{a}}\end{array}\end{array}$ & $\begin{array}{c}347.9(241.9-588.1) \\
n=10\end{array}$ \\
\hline & $N A(n g / g)$ & $\begin{array}{c}67.4(54.7-395.9) \\
n=8\end{array}$ & $\begin{array}{c}76.2(61.0-127.9) \\
n=8^{b}\end{array}$ & $\begin{array}{c}36.2(26.6-48.9) \\
n=10^{b}\end{array}$ & $\begin{array}{c}60.2(46.4-81.5) \\
n=10\end{array}$ \\
\hline \multirow[t]{5}{*}{ hippocampus } & 5-HIAA (ng/g) & $\begin{array}{c}383.2(260.5-689.9) \\
n=10\end{array}$ & $\begin{array}{c}385.0(348.7-954.9) \\
n=9^{b}\end{array}$ & $\begin{array}{c}271.9(239.1-331.6) \\
n=10^{b}\end{array}$ & $\begin{array}{c}284.4(242.9-349.3) \\
n=10\end{array}$ \\
\hline & $\mathrm{DA}(\mathrm{ng} / \mathrm{g})$ & $\begin{array}{c}9.0(5.2-120.8) \\
n=9^{\mathrm{a}}\end{array}$ & $\begin{array}{c}5.9 \begin{array}{l}(4.7-16.7) \\
n=9\end{array}\end{array}$ & $\begin{array}{c}3.8(2.4-5.3) \\
n=10^{\mathrm{a}}\end{array}$ & $\begin{array}{c}8.9(6.5-23.7) \\
n=10\end{array}$ \\
\hline & HVA (ng/g) & $\begin{array}{c}224.5(157.6-269.6) \\
n=10\end{array}$ & $\begin{array}{c}206.5(132.0-249.9) \\
n=9\end{array}$ & $\begin{array}{c}133.0(108.7-168.0) \\
n=10^{f}\end{array}$ & $\begin{array}{c}236.1 \begin{array}{l}(193.3-333.1) \\
n=10^{f}\end{array}\end{array}$ \\
\hline & 5-HIAA/5-HT & $\begin{array}{c}8.2(5.5-9.6) \\
n=10^{\mathrm{a}}\end{array}$ & $\begin{array}{c}6.2(4.2-13.8) \\
n=9\end{array}$ & $\begin{array}{c}3.8(2.8-5.2) \\
\mathrm{n}=10^{\mathrm{a}}\end{array}$ & $\begin{array}{c}4.4(3.4-5.9) \\
\quad n=10\end{array}$ \\
\hline & DOPAC/DA & $\begin{array}{c}0.4(0.1-1.7) \\
n=9^{\mathrm{a}}\end{array}$ & $\begin{array}{c}1.5(0.5-2.3) \\
n=9\end{array}$ & $\begin{array}{l}3.0(1.6-3.7) \\
n=10^{\mathrm{a}, \mathrm{ff}}\end{array}$ & $\begin{array}{c}0.7(0.4-1.2) \\
n=10^{f f}\end{array}$ \\
\hline \multirow[t]{5}{*}{ thalamus } & MHPG (ng/g) & $\begin{array}{c}1,273.7(776.8-1,740.1) \\
n=10^{\text {aa }}\end{array}$ & $\begin{array}{c}686.2(521.1-1,585.9) \\
n=10\end{array}$ & $\begin{array}{c}248.5(174.0-568.9) \\
n=10^{\text {aa }}\end{array}$ & $\begin{array}{c}494.4(315.2-1,125.7) \\
n=10\end{array}$ \\
\hline & $N A(n g / g)$ & $\begin{array}{c}140.5(111.1-164.6) \\
n=8^{\mathrm{a}}\end{array}$ & $\begin{array}{c}183.9(146.7-261.6) \\
n=10^{b}\end{array}$ & $\begin{array}{l}47.0(25.0-76.6) \\
n=10^{\mathrm{a}, \mathrm{b}}, \mathrm{ff}\end{array}$ & $\begin{array}{c}162.9(91.6-213.7) ; \\
n=10^{2 f f}\end{array}$ \\
\hline & $\mathrm{DA}(\mathrm{ng} / \mathrm{g})$ & $\begin{array}{c}14.0(8.6-31.6) \\
n=10^{\text {aaa }}\end{array}$ & $\begin{array}{c}13.1(9.3-28.5) \\
n=10^{\mathrm{bb}}\end{array}$ & $\begin{array}{c}1.4(1.0-5.3) ; \\
n=10^{\text {aaa, }} \text { bb, fff }\end{array}$ & $\begin{array}{c}19.7(8.7-31.8) \\
n=10^{\text {fff }}\end{array}$ \\
\hline & DOPAC/DA & $\begin{array}{c}1.0(0.5-1.3) \\
n=10^{\mathrm{a}}\end{array}$ & $\begin{array}{c}1.0(0.5-1.6) \\
n=10^{b}\end{array}$ & $\begin{array}{c}3.7(1.9-11.3) \\
n=10^{a, b}, f^{\prime}\end{array}$ & $\begin{array}{c}0.9(0.5-1.1) \\
n=10^{f f}\end{array}$ \\
\hline & HVA/DA & $\begin{array}{c}31.0(20.7-42.2) \\
n=10^{\mathrm{a}}\end{array}$ & $\begin{array}{c}29.2(14.8-70.0) \\
n=10^{b}\end{array}$ & $\begin{array}{c}225.5(55.8-361.9) \\
n=10^{a, b}, f\end{array}$ & $\begin{array}{c}29.2(23.1-44.3) \\
n=10^{f}\end{array}$ \\
\hline \multirow[t]{4}{*}{ BA11 } & 5-HIAA (ng/g) & $\begin{array}{c}238.6(165.2-464.6) \\
n=9\end{array}$ & $\begin{array}{c}293.9(176.1-424.6) \\
n=10^{b}\end{array}$ & $\begin{array}{c}83.9(65.9-132.3) \\
n=10^{\mathrm{b}, \mathrm{f}}\end{array}$ & $\begin{array}{c}222.3(161.4-353.6) \\
n=10^{f}\end{array}$ \\
\hline & HVA (ng/g) & $\begin{array}{c}146.1 \begin{array}{l}(92.7-165.2) \\
n=9^{\mathrm{a}}\end{array}\end{array}$ & $\begin{array}{c}132.9(84.3-182.5) \\
n=10^{b}\end{array}$ & $\begin{array}{c}62.5(43.4-83.7) \\
n=10^{a, b}, \mathrm{f}\end{array}$ & $\begin{array}{c}118.9(91.7-166.1) ; \\
n=10^{f}\end{array}$ \\
\hline & 5-HT (ng/g) & $\begin{array}{c}11.8(6.8-20.2) \\
n=9\end{array}$ & $\begin{array}{c}17.3(10.5-36.7) \\
n=10^{\mathrm{bb}}\end{array}$ & $\begin{array}{c}5.9(3.7-8.2) \\
\mathrm{n}=10^{\mathrm{bb}}\end{array}$ & $\begin{array}{c}9.0(5.6-14.5) \\
n=10\end{array}$ \\
\hline & MHPG/NA & $\begin{array}{c}81.7(37.6-111.1) ; \\
n=6^{a}\end{array}$ & $\begin{array}{c}47.1(21.3-64.3) \\
n=10\end{array}$ & $\begin{array}{c}21.5(10.2-31.5) \\
n=10^{a}\end{array}$ & $\begin{array}{c}42.7 \begin{array}{c}(33.1-69.9) \\
n=10\end{array}\end{array}$ \\
\hline
\end{tabular}


Table 2 Focus depression: comparison of the brain monoamine levels between AD + D, AD-D, DLB + D and CONTR (Continued)

\begin{tabular}{|c|c|c|c|c|c|}
\hline \multirow[t]{4}{*}{ BA22 } & MHPG (ng/g) & $\begin{array}{c}779.9(546.3-1,177.4) \\
n=10^{\text {aa }}\end{array}$ & $\begin{array}{c}810.3(308.6-1,492.7) \\
n=10\end{array}$ & $\begin{array}{c}296.0(171.6-521.2) \\
n=10^{\text {aa }}\end{array}$ & $\begin{array}{c}609.6(419.2-1,591.4) \\
n=10\end{array}$ \\
\hline & 5-HIAA (ng/g) & $\begin{array}{c}472.3(293.2-793.5) \\
n=10^{\text {aaa }}\end{array}$ & $\begin{array}{c}311.3(166.6-958.3) \\
n=10^{b}\end{array}$ & $\begin{array}{c}64.4(46.5-133.3) \\
\quad n=10^{\text {aaa, }}, \mathrm{f}\end{array}$ & $\begin{array}{c}255.2(127.0-473.0) \\
n=10^{f}\end{array}$ \\
\hline & HVA (ng/g) & $\begin{array}{c}138.4(125.5-207.8) \\
n=10^{a}\end{array}$ & $\begin{array}{c}116.8(91.4-148.4) ; \\
n=10^{b}\end{array}$ & $\begin{array}{c}61.0(47.1-89.0) \\
n=10^{\text {a, }}, \mathrm{f}\end{array}$ & $\begin{array}{c}141.7(86.0-240.0) ; \\
n=10^{f}\end{array}$ \\
\hline & MHPG/NA & $\begin{array}{c}83.5(23.6-92.1) \\
n=7\end{array}$ & $\begin{array}{c}46.5(29.1-137.8) \\
n=9^{b}\end{array}$ & $\begin{array}{c}17.9(9.0-29.5) \\
n=10^{b}\end{array}$ & $\begin{array}{c}33.9(22.1-79.3) \\
n=10\end{array}$ \\
\hline \multirow[t]{3}{*}{ BA17 } & 5-HIAA (ng/g) & $\begin{array}{c}143.6(98.1-191.0) ; \\
n=9^{\text {aa }}\end{array}$ & $\begin{array}{c}117.8(92.1-187.9) ; \\
n=10^{b}\end{array}$ & $\begin{array}{l}47.8(32.6-69.5) \\
n=10^{a a, b, ~ f f ~}\end{array}$ & $\begin{array}{c}147.9(118.4-198.0) \\
n=10^{\text {ff }}\end{array}$ \\
\hline & $\mathrm{DA}(\mathrm{ng} / \mathrm{g})$ & $\begin{array}{c}12.2(7.1-17.9) \\
n=9^{d, c}\end{array}$ & $\begin{array}{c}2.7(1.5-3.8) ; \\
n=10^{c}\end{array}$ & $\begin{array}{c}4.3(2.6-7.1) \\
n=10\end{array}$ & $\begin{array}{l}3.5(1.6-4.8) \\
n=10^{d}\end{array}$ \\
\hline & 5-HT (ng/g) & $\begin{array}{c}13.7(6.5-24.1) \\
n=9^{\mathrm{a}}\end{array}$ & $\begin{array}{c}12.7(3.4-24.4) \\
n=10\end{array}$ & $\begin{array}{l}2.6(2.2-5.7) \\
n=10^{a, f}\end{array}$ & $\begin{array}{c}8.8(5.5-20.3) \\
n=10^{f}\end{array}$ \\
\hline \multirow[t]{4}{*}{ Cerebellum } & 5-HIAA (ng/g) & $\begin{array}{c}244.3(103.4-538.2) \\
n=9^{a}\end{array}$ & $\begin{array}{c}276.3(117.6-465.3) \\
n=9^{b}\end{array}$ & $\begin{array}{c}57.3(38.4-81.8) \\
n=10^{a, b, f}\end{array}$ & $\begin{array}{c}181.9(103.3-333.6) ; \\
n=10^{f}\end{array}$ \\
\hline & $\mathrm{DA}(\mathrm{ng} / \mathrm{g})$ & $\begin{array}{c}5.6(2.4-7.1) \\
n=9^{a}\end{array}$ & $\begin{array}{c}3.0(2.0-17.9) \\
n=9\end{array}$ & $\begin{array}{c}2.3(0.5-2.6) \\
n=10^{\mathrm{a}}\end{array}$ & $\begin{array}{c}3.4(2.2-4.7) \\
n=10\end{array}$ \\
\hline & HVA (ng/g) & $\begin{array}{c}105.4 \begin{array}{l}(51.1-137.6) \\
n=9^{a}\end{array}\end{array}$ & $\begin{array}{c}64.5(55.9-94.9) \\
n=9^{b}\end{array}$ & $\begin{array}{c}35.3(22.9-59.5) \\
n=10^{\mathrm{a}, \mathrm{b}}\end{array}$ & $\begin{array}{c}94.5(43.1-149.2) ; \\
n=10\end{array}$ \\
\hline & DOPAC/DA & $\begin{array}{c}2.3(1.0-5.8) \\
n=9\end{array}$ & $\begin{array}{c}1.7(0.3-3.9) \\
n=9^{b}\end{array}$ & $\begin{array}{c}6.7(3.7-13.9) \\
n=10^{b}\end{array}$ & $\begin{array}{c}1.4(0.7-6.0) \\
n=9\end{array}$ \\
\hline \multirow[t]{5}{*}{ Locus coeruleus } & DOPAC (ng/g) & $\begin{array}{c}55.5 \begin{array}{c}(32.8-102.6) \\
n=9^{\mathrm{a}}\end{array}\end{array}$ & $\begin{array}{c}41.5(28.1-101.6) ; \\
n=9\end{array}$ & $\begin{array}{l}19.3(12.7-28.1) \\
n=10^{\mathrm{a}, \mathrm{ff}}\end{array}$ & $\begin{array}{c}78.8(47.1-130.3) \\
n=10^{f f}\end{array}$ \\
\hline & $\mathrm{DA}(\mathrm{ng} / \mathrm{g})$ & $\begin{array}{c}41.0 \begin{array}{c}(25.9-102.9) \\
n=9^{\mathrm{a}}\end{array}\end{array}$ & $\begin{array}{c}34.5(20.0-49.4) \\
n=9\end{array}$ & $\begin{array}{c}15.8(7.0-25.0) \\
n=10^{a, f}\end{array}$ & $\begin{array}{c}37.1(30.1-55.7) \\
n=10^{f}\end{array}$ \\
\hline & HVA (ng/g) & $\begin{array}{c}1,361.6(963.2-1,904.9) ; \\
n=9^{a}\end{array}$ & $\begin{array}{c}1,082.7(834.7-1,634.6) ; \\
n=9^{b}\end{array}$ & $\begin{array}{l}540.2(378.5-887.8) \\
\quad n=10^{\mathrm{a}, \mathrm{b}, \mathrm{fff}}\end{array}$ & $\begin{array}{c}1,603.2(1,284.7-1,964.1) ; \\
n=10^{\text {fff }}\end{array}$ \\
\hline & HVA/5-HIAA & $\begin{array}{l}0.4(0.2-0.5) \\
n=9\end{array}$ & $\begin{array}{c}0.2(0.2-0.3) \\
n=9\end{array}$ & $\begin{array}{c}0.2(0.1-0.2) \\
n=10^{f}\end{array}$ & $\begin{array}{c}0.4(0.3-0.5) \\
n=10^{f}\end{array}$ \\
\hline & MHPG/NA & $\begin{array}{c}1.1(0.9-2.6) \\
n=9\end{array}$ & $\begin{array}{c}1.5(1.3-2.0) \\
n=9^{b}\end{array}$ & $\begin{array}{c}3.9(2.1-4.8) \\
n=10^{b}\end{array}$ & $\begin{array}{c}0.8(0.5-2.4) \\
n=10\end{array}$ \\
\hline
\end{tabular}

Median (IQR); Kruskal-Wallis analyses $(P<0.05)$ with post-hoc Mann-Whitney $\mathrm{U}$ tests were performed; only data remaining statistically significant following Bonferroni correction for multiple comparisons $(P<0.00833$; one superscript letter) are presented above; significant differences with $P<0.001$ and $P<0.0001$ are respectively indicated with two and three repeated superscript letters; the following letters are used: ${ }^{\mathrm{a}} A D+D$ vs. $D L B+D,{ }^{b} A D-D$ vs. $D L B+D,{ }^{c} A D+D$ vs. $A D-D,{ }^{d} A D+D$ vs. CONTR, ${ }^{\mathrm{e}} \mathrm{AD}-\mathrm{D}$ vs. CONTR and $\mathrm{f} D L B+\mathrm{D}$ vs. CONTR.

5-HIAA, 5-hydroxyindoleacetic acid; 5-HT, 5-hydroxytryptamine (serotonin); AD + D/-D, depressed/nondepressed Alzheimer's disease patients; BA, Brodmann area; CONTR, control subjects; DA, dopamine; DLB + D, depressed dementia with Lewy bodies patients; DOPAC, 3,4-dihydroxyphenylacetic acid; HVA, homovanillic acid; MA and MT, monoamines and metabolites; MHPG, 3-methoxy-4-hydroxyphenylglycol; NA, noradrenaline.

compared to the $\mathrm{AD}+\mathrm{D}(P=0.003,0.003$ and 0.001 , respectively) and control group $(P=0.0001,0.002$ and 0.00001 , respectively) (Table 2 ).

Finally, DA levels of BA17 were significantly increased in $\mathrm{AD}+\mathrm{D}$ patients compared to $\mathrm{AD}-\mathrm{D}$ patients and the control group ( $P=0.003$ and 0.006 , respectively), whereas 5 -HT levels of BA9 were significantly higher in AD-D patients compared to the control subjects $(P=0.007)$.

MHPG levels across different postmortem brain regions in DLB versus $A D$ and controls

Remarkably, MHPG was significantly decreased in seven out of eleven postmortem brain regions of DLB subjects compared to $\mathrm{AD}+\mathrm{D}$ and/or $\mathrm{AD}-\mathrm{D}$ patients, that is, BA9 $(P=0.004$ compared to AD-D), BA10 $(P=0.04$ and
0.008 compared to $\mathrm{AD}+\mathrm{D}$ and $\mathrm{AD}-\mathrm{D}$, respectively), amygdala $(P=0.007$ compared to $\mathrm{AD}+\mathrm{D})$, hippocampus $(P=0.023$ compared to $\mathrm{AD}+\mathrm{D})$, thalamus $(P=0.0002$ and 0.013 compared to $\mathrm{AD}+\mathrm{D}$ and $\mathrm{AD}-\mathrm{D}$, respectively), BA11 $(P=0.028$ compared to AD-D) and BA22 $(P=0.0004$ and 0.041 compared to $\mathrm{AD}+\mathrm{D}$ and $\mathrm{AD}-\mathrm{D}$, respectively) (Figure 1). As for BA24, MHPG levels were almost significantly lower in DLB patients compared to both $\mathrm{AD}+\mathrm{D}$ and AD-D subjects (borderline significance; $P=0.059$ for both). Surprisingly, in BA17, DLB patients as well as control subjects had significantly higher MHPG levels compared to their $\mathrm{AD}+\mathrm{D}$ counterparts $(P=0.011$ for both). In total, for five out of seven brain regions in which statistically different MHPG levels were found $(P<0.05)$, these differences remained significant following 
Table 3 Focus psychosis: comparison of the brain monoamine levels between $A D+D+P, A D+D-P, D L B+D+P$ and DLB + D-P

\begin{tabular}{|c|c|c|c|c|c|}
\hline Brain region & $\begin{array}{l}\text { MA and MT } \\
\text { or ratio }\end{array}$ & $\begin{array}{l}A D+D+P \\
\text { (number }=5 \text { ) }\end{array}$ & $\begin{array}{l}A D+D-P \\
(\text { number }=5)\end{array}$ & $\begin{array}{l}\text { DLB + D + P } \\
\text { (number = 5) }\end{array}$ & $\begin{array}{l}\text { DLB + D-P } \\
\text { (number = 5) }\end{array}$ \\
\hline \multirow[t]{2}{*}{ BA9 } & HVA (ng/g) & $146.6(85.1-198.0) ; n=4$ & $127.2(117.8-152.5) ; n=5^{b}$ & $110.7(81.8-156.2) ; n=5$ & $51.5(44.1-73.0) ; n=5^{b}$ \\
\hline & $5-\mathrm{HT}(\mathrm{ng} / \mathrm{g})$ & $5.3(3.3-15.8) ; n=4$ & $14.1(8.7-76.8) ; n=5^{b, d}$ & $4.2(1.7-5.2) ; n=5^{d}$ & $3.4(2.6-3.5) ; n=5^{b}$ \\
\hline \multirow[t]{3}{*}{ BA10 } & 5-HIAA (ng/g) & $227.6(99.6-331.1) ; n=5$ & $250.9(169.7-441.7) ; n=5^{d}$ & $70.0(56.1-128.9) ; n=5^{d}$ & $87.7(63.2-180.0) ; n=5$ \\
\hline & HVA (ng/g) & $111.3(84.7-131.7) ; n=5^{c}$ & $117.9(95.0-139.2) ; n=5^{b}$ & $62.1(59.2-104.8) ; n=5$ & $45.0(31.9-54.4) ; n=5^{c, b}$ \\
\hline & $5-H \mathrm{~T}(\mathrm{ng} / \mathrm{g})$ & $16.0(10.4-35.8) ; n=5^{a, c}$ & $16.1(10.8-161.0) ; n=5^{b, d}$ & $4.6(1.6-5.6) ; n=5^{a, d}$ & $4.1(2.9-6.0) ; n=5^{c, b}$ \\
\hline \multirow[t]{2}{*}{ BA24 } & HVA (ng/g) & $212.8(163.4-265.0) ; n=5$ & $216.2(185.3-263.1) ; n=5^{b}$ & $167.7(131.2-206.1) ; n=5$ & $102.9(79.6-140.2) ; n=5^{b}$ \\
\hline & $5-\mathrm{HT}(\mathrm{ng} / \mathrm{g})$ & $32.2(29.3-48.7) ; n=5$ & $47.0(39.4-165.6) ; n=5^{d}$ & $18.9(7.1-24.6) ; n=5^{d}$ & $22.0(14.9-32.0) ; n=5$ \\
\hline \multirow[t]{2}{*}{ Hippocampus } & MHPG (ng/g) & $459.5(224.9-1,000.7) ; n=5$ & $1,078.1(850.3-1,099.3) ; n=5^{d}$ & $358.9(171.2-569.5) ; n=5^{d}$ & $383.1(318.3-570.7) ; n=5$ \\
\hline & HVA (ng/g) & $193.9(146.5-462.3) ; n=5$ & $245.6(174.4-284.7) ; n=5^{b}$ & $143.3(114.4-250.4) ; n=5$ & $116.9(106.4-138.6) ; n=5^{b}$ \\
\hline \multirow[t]{3}{*}{ Thalamus } & MHPG (ng/g) & $793.0(678.3-1,344.8) ; n=5$ & $1,342.5(1,273.7-1,860.6) ; n=5^{b, d}$ & 245.5 (143.1-569.9); $n=5^{d}$ & $251.6(164.9-697.1) ; n=5^{b}$ \\
\hline & $\mathrm{DA}(\mathrm{ng} / \mathrm{g})$ & $17.0(7.1-22.7) ; n=5$ & $11.1(9.3-857.5) ; n=5^{b}$ & $1.1(1.0-5.3) ; n=5$ & $1.7(0.8-5.6) ; n=5^{b}$ \\
\hline & HVA/DA & $32.0(25.5-53.1) ; n=5$ & $30.1(5.7-42.4) ; n=5^{d}$ & $230.5(146.2-382.8) ; n=5^{d}$ & $137.7(26.5-439.3) ; n=5$ \\
\hline \multirow[t]{2}{*}{ BA22 } & 5-HIAA (ng/g) & $756.7(241.2-1,147.4) ; n=5$ & $450.2(332.4-507.9) ; n=5^{b}$ & $64.0(44.1-162.6) ; n=5$ & $81.3(49.0-158.8) ; n=5^{b}$ \\
\hline & HVA (ng/g) & $181.7(135.6-218.4) ; n=5^{a, c}$ & $129.5(80.6-178.2) ; n=5$ & $72.0(61.0-104.0) ; n=5^{a}$ & $50.8(31.3-69.5) ; n=5^{c}$ \\
\hline \multirow[t]{2}{*}{ BA17 } & 5-HIAA (ng/g) & $138.3(93.9-189.9) ; n=4$ & $143.6(98.1-215.2) ; n=5^{d}$ & $43.1(22.4-60.3) ; n=5^{d}$ & $54.7(38.4-110.8) ; n=5$ \\
\hline & $5-\mathrm{HT}(\mathrm{ng} / \mathrm{g})$ & $9.8(4.2-19.5) ; n=4$ & $14.5(7.4-26.8) ; n=5^{b}$ & $2.5(1.6-5.2) ; n=5$ & $3.5(2.5-5.9) ; n=5^{b}$ \\
\hline \multirow{3}{*}{$\begin{array}{l}\text { Locus } \\
\text { coeruleus }\end{array}$} & DA (ng/g) & $32.3(20.2-132.4) ; n=5^{c}$ & $52.5(33.0-102.5) ; n=4$ & $21.1(12.0-42.1) ; n=5$ & $12.1(6.5-16.5) ; n=5^{c}$ \\
\hline & HVA (ng/g) & $\begin{array}{c}1,361.6(963.2-1,908.4) \\
n=5^{c}\end{array}$ & $\begin{array}{c}1,225.4(867.6-1,834.0) \\
n=4\end{array}$ & $\begin{array}{c}884.8(506.1-1,013.5) \\
n=5\end{array}$ & $\begin{array}{c}408.0(319.8-648.7) \\
n=5^{c}\end{array}$ \\
\hline & $\mathrm{HVA} / 5-\mathrm{HIAA}$ & $0.3(0.2-0.6) ; n=5^{c}$ & $0.4(0.2-0.5) ; n=4$ & $0.2(0.2-0.4) ; n=5$ & $0.1(0.08-0.2) ; n=5^{c}$ \\
\hline
\end{tabular}

Median (IQR); Kruskal-Wallis analyses $(P<0.05)$ with post-hoc Mann-Whitney $\mathrm{U}$ tests were performed; only data remaining statistically significant following Bonferroni correction for multiple comparisons $\left(P<0.00833\right.$; one superscript letter) are presented above; the following letters are used: ${ }^{a} A D+D+P$ vs. $D L B+D+P$, ${ }^{b} A D+D-P$ vs. $D L B+D-P,{ }^{c} A D+D+P$ vs. DLB + D-P and ${ }^{d} A D+D-P$ vs. DLB + D + P.

5-HIAA, 5-hydroxyindoleacetic acid; 5-HT, 5-hydroxytryptamine (serotonin); $A D+D+P /-P$, psychotic/nonpsychotic Alzheimer's disease patients (within the depressed AD group); BA, Brodmann area; DA, dopamine; DLB + D + P/-P, psychotic/nonpsychotic dementia with Lewy bodies patients (within the depressed DLB group); HVA, homovanillic acid; MA and MT, monoamines and metabolites; MHPG, 3-methoxy-4-hydroxyphenylglycol.

Bonferoni correction for multiple comparisons (Table 2; Figure 1).

For the cerebellar cortex and the LC, no MHPG differences were spotted between the DLB and AD groups, except for NA levels and MHPG/NA ratios in the LC of DLB patients, which were respectively significantly decreased and increased compared to both $A D+D$ and AD-D patients (for AD + D: $P=0.041$ for both; for AD-D: $P=0.018$ and 0.007 (Table 2)).

Besides the DLB-AD comparison, MHPG concentrations were significantly increased in the $A D+D$ and/or $A D-D$ group(s) compared to the control group as well, that is, for BA9 $(P=0.049$ compared to AD-D), BA10 $(P=0.04$ and 0.01 compared to $\mathrm{AD}+\mathrm{D}$ and $\mathrm{AD}-\mathrm{D}$, respectively), BA24 $(P=0.03$ and 0.049 compared to $\mathrm{AD}+\mathrm{D}$ and $\mathrm{AD}-\mathrm{D}$, respectively), amygdala $(P=0.027$ compared to $\mathrm{AD}+\mathrm{D})$, hippocampus $(P=0.027$ compared to $\mathrm{AD}+\mathrm{D})$ and thalamus $(P=0.016$ compared to $\mathrm{AD}+\mathrm{D})$. As for BA17, MHPG levels of the control group were significantly increased compared to the $\mathrm{AD}+\mathrm{D}$ group $(P=0.011)$. Finally, in BA11, BA22 and cerebellar cortex, we also noticed significantly lower MHPG levels in DLB patients compared to control subjects ( $P=0.007,0.011$ and 0.023 , respectively) (Figure 1).

Focus psychosis: neurochemical comparison of brain monoamines, metabolites and ratios between $A D+D+P$, $A D+D-P, D L B+D+P$ and DLB + D-P

Table 3 summarizes the brain monoaminergic differences between the subgroups of psychotic and nonpsychotic AD and DLB patients that remained statistically significant following Bonferroni correction for multiple comparisons $(P<0.00833)$. Nonsignificant data were excluded from the Table.

With regard to the $\mathrm{DLB}+\mathrm{D}+\mathrm{P}$ and $\mathrm{AD}+\mathrm{D}+\mathrm{P}$ subgroups, statistical analyses showed that 5-HT levels of BA10, as well as HVA levels of BA22 were significantly lower in $\mathrm{DLB}+\mathrm{D}+\mathrm{P}$ patients compared to $\mathrm{AD}+\mathrm{D}+\mathrm{P}$ patients $(P=0.008$ for both). Similarly, 5-HT levels of BA9, BA10 and BA17, as well as 5-HIAA levels of BA22 and HVA levels of BA9, BA10, BA24 and the 
Table 4 Significant brain monoaminergic correlates of NPS in DLB and AD

\begin{tabular}{|c|c|c|c|c|}
\hline Study group & Brain region & MA and MT or ratio & NPS feature & Spearman's Rank Order correlation \\
\hline \multicolumn{5}{|l|}{ DLB patients } \\
\hline \multicolumn{5}{|l|}{ Number $=10$} \\
\hline & \multirow[t]{2}{*}{ BA10 } & \multirow[t]{2}{*}{ DA (ng/g tissue) } & CMAI cluster 3 & \multirow[t]{2}{*}{$R=+0.841, P=0.002$, number $=10$} \\
\hline & & & verbal agitation & \\
\hline & \multirow[t]{3}{*}{ hippocampus } & \multirow[t]{2}{*}{ DA (ng/g tissue) } & Behave-AD cluster $A B$ & \multirow[t]{2}{*}{$R=+0.928, P=0.0001$, number $=10$} \\
\hline & & & psychosis & \\
\hline & & DA (ng/g tissue) & Behave-AD total score & $R=+0.825, P=0.003$, number $=10$ \\
\hline & \multirow[t]{4}{*}{ thalamus } & \multirow[t]{2}{*}{ DA (ng/g tissue) } & CSDD total scores & \multirow[t]{2}{*}{$R=+0.839, P=0.002$, number $=10$} \\
\hline & & & depression & \\
\hline & & \multirow[t]{2}{*}{ HVA/DA ratio } & CSDD total scores & \multirow[t]{2}{*}{$R=-0.851, P=0.002$, number $=10$} \\
\hline & & & depression & \\
\hline & \multirow[t]{2}{*}{ BA22 } & \multirow[t]{2}{*}{ DA (ng/g tissue) } & CMAl total score & \multirow[t]{2}{*}{$R=+0.827, P=0.003$, number $=10$} \\
\hline & & & agitation & \\
\hline & \multirow[t]{2}{*}{ cerebellum } & \multirow[t]{2}{*}{ DOPAC/DA ratio } & Behave-AD cluster F & \multirow[t]{2}{*}{$R=-0.846, P=0.002$, number $=10$} \\
\hline & & & affective disturbances & \\
\hline
\end{tabular}

\section{AD patients}

Number $=20$

\begin{tabular}{|c|c|c|c|}
\hline \multirow[t]{4}{*}{ amygdala } & DA (ng/g tissue) & $\begin{array}{l}\text { Behave-AD cluster AB } \\
\text { psychosis }\end{array}$ & $R=-0.728, P=0.0009$, number $=17$ \\
\hline & HVA/DA ratio & $\begin{array}{l}\text { Behave-AD cluster AB } \\
\text { psychosis }\end{array}$ & $R=+0.766, P=0.0003$, number $=17$ \\
\hline & HVA/DA ratio & $\begin{array}{l}\text { CMAl cluster } 3 \\
\text { verbal agitation }\end{array}$ & $R=+0.786, P=0.0002$, number $=17$ \\
\hline & HVA/DA ratio & $\begin{array}{l}\text { CMAl total score } \\
\text { agitation }\end{array}$ & $R=+0.671, P=0.003$, number $=17$ \\
\hline hippocampus & HVA/5-HIAA ratio & $\begin{array}{l}\text { Behave-AD cluster } C \\
\text { activity disturbances }\end{array}$ & $R=+0.659, P=0.002$, number $=19$ \\
\hline \multirow[t]{2}{*}{ BA17 } & HVA/DA ratio & $\begin{array}{l}\text { Behave-AD cluster F } \\
\text { affective disturbances }\end{array}$ & $R=-0.670, P=0.002$, number $=19$ \\
\hline & DA (ng/g tissue) & $\begin{array}{l}\text { CSDD total scores } \\
\text { depression }\end{array}$ & $R=+0.662, P=0.002$, number $=19$ \\
\hline LC & MHPG/NA ratio & $\begin{array}{l}\text { Behave-AD cluster } \mathrm{D} \\
\text { agitation and aggression }\end{array}$ & $R=+0.727, P=0.001$, number $=16$ \\
\hline
\end{tabular}

Significant correlations between NPS and neurochemical data in postmortem brain tissue samples of DLB and AD patients as indicated by Spearman's Rank Order correlation statistics (correlation coefficient $(R), P$ value and sample size $(n)$ ). Only correlations remaining statistically significant following Bonferroni correction are displayed above $(P<0.0033)$. The four most significant correlations $(P<0.001)$ are shown in bold. 5-HIAA, 5-hydroxy-3-indoleacetic acid; AD, Alzheimer's disease; BA, Brodmann area; Behave-AD, Behavioral Pathology in Alzheimer's Disease Rating Scale; CMAl, Cohen-Mansfield Agitation Inventory; CSDD, Cornell Scale for Depression in Dementia; DA, dopamine; DLB, dementia with Lewy bodies; DOPAC, 3,4-dihydroxyphenylacetic acid; HVA, homovanillic acid; LC, locus coeruleus; MA and MT, monoamines and metabolites; MFS, Middelheim Frontality Score; MHPG, 3-methoxy-4-hydroxyphenylglycol; NA, noradrenaline; NPS, neuropsychiatric symptoms.

hippocampus, were all significantly lower in DLB $+\mathrm{D}-\mathrm{P}$ patients compared to AD $+\mathrm{D}-\mathrm{P}$ patients $(P=0.008$ for all; Table 3).

Notably, in the amygdala, DOPAC and DA levels, as well as HVA/DA ratios, were significantly decreased and increased, respectively, in $\mathrm{AD}+\mathrm{D}+\mathrm{P}$ patients compared to $\mathrm{AD}+\mathrm{D}-\mathrm{P}$ patients, although significance was not maintained following a Bonferroni correction for multiple comparisons (data not shown; for DOPAC and DA: $P=0.032$ and 0.014 ; for HVA/DA: $P=0.016$ ). The same applies for the monoaminergic group comparison of $\mathrm{DLB}+\mathrm{D}+\mathrm{P} /-\mathrm{P}$, with significantly increased HVA levels in BA9, BA10, BA11, BA22 and BA24, as well as increased DA levels in the hippocampus and increased 


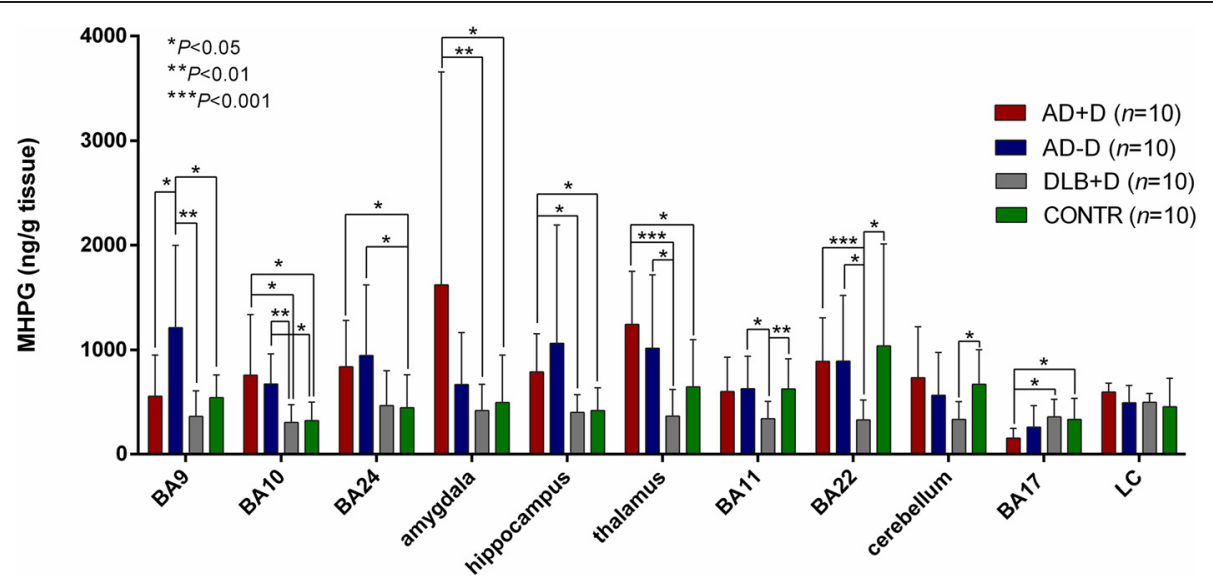

Figure 1 MHPG concentrations across different brain regions in DLB + D compared to AD + D, AD-D and CONTR. Data are presented as mean with SD. Nonparametric Mann-Whitney U statistics were performed. MHPG levels of seven out of eleven postmortem brain regions were significantly decreased in DLB $+D$ compared to $A D+D$ and/or AD-D patients ( $P$ values vary from $<0.05$ to $<0.001$ ). In BA17, MHPG concentrations of $D L B+D$ patients were significantly higher. AD + D/-D, depressed/nondepressed Alzheimer's disease patients; BA, Brodmann area; CONTR, control subjects; DLB + D, depressed dementia with Lewy bodies patients; LC, locus coeruleus; MHPG, 3-methoxy-4-hydroxyphenylglycol.

HVA/5-HIAA ratios in the LC, indicating the inhibitory effect of the serotonergic system on dopaminergic neurotransmission $[62,63]$ of DLB $+\mathrm{D}+\mathrm{P}$ patients compared to DLB +D-P patients (data not shown; for $\mathrm{HVA}_{\mathrm{BA} 9 / \mathrm{BA} 10 / \mathrm{BA} 11}$ and $\mathrm{HVA} / 5-\mathrm{HIAA}_{\mathrm{LC}}: P=0.016$; for HVA $_{\text {BA22/BA24: }} P=0.047$; for $\mathrm{DA}_{\text {hippocampus }}: P=0.032$ ).

\section{Brain monoaminergic correlates of NPS features in the DLB and AD population}

Table 4 shows the significant brain monoaminergic correlates of NPS in the DLB and total AD group, only for those that remained significant following Bonferroni correction. Both $\mathrm{AD}$ subgroups were joined into one total AD group $(n=20)$ in order to obtain a heterogeneous cohort with a better distribution of NPS scores since $\mathrm{AD}+\mathrm{D}$ patients had much higher behavioral scores compared to AD-D patients (Table 1). Furthermore, there were no significant brain monoamine differences between $\mathrm{AD}+\mathrm{D}$ and $\mathrm{AD}-\mathrm{D}$ subjects, except of $\mathrm{DA}$ levels in
BA17 (Table 2). The merging also resulted in increased statistical power. The four most significant correlations are described below.

In the DLB group, hippocampal DA levels strongly correlated with Behave-AD cluster $\mathrm{AB}$ scores (psychosis) $(P=0.0001, R=+0.928, \mathrm{n}=10$; Figure $2 \mathrm{~B})$.

In the total $\mathrm{AD}$ population, HVA/DA ratios of the amygdala strongly correlated with Behave-AD cluster $\mathrm{AB}$ scores (psychosis) $(P=0.0003, R=+0.766, \mathrm{n}=17$; Figure $2 \mathrm{C}$ ) and CMAI cluster 3 scores (verbal agitation) $(P=0.0002, R=+0.786, \mathrm{n}=17$; Figure $2 \mathrm{~A})$, whereas DA levels of the amygdala inversely correlated with Behave$\mathrm{AD}$ cluster $\mathrm{AB}$ scores (psychosis) $(P=0.0009, R=-0.728$, $\mathrm{n}=17$; Figure 2D). Noteworthy, MHPG/NA ratios of the LC significantly correlated with Behave-AD cluster $\mathrm{D}$ scores (agitation/aggression) in the total $\mathrm{AD}$ group as well ( $P=0.001, R=+0.728, \mathrm{n}=16)$, although statistical significance was not maintained after Bonferroni correction.

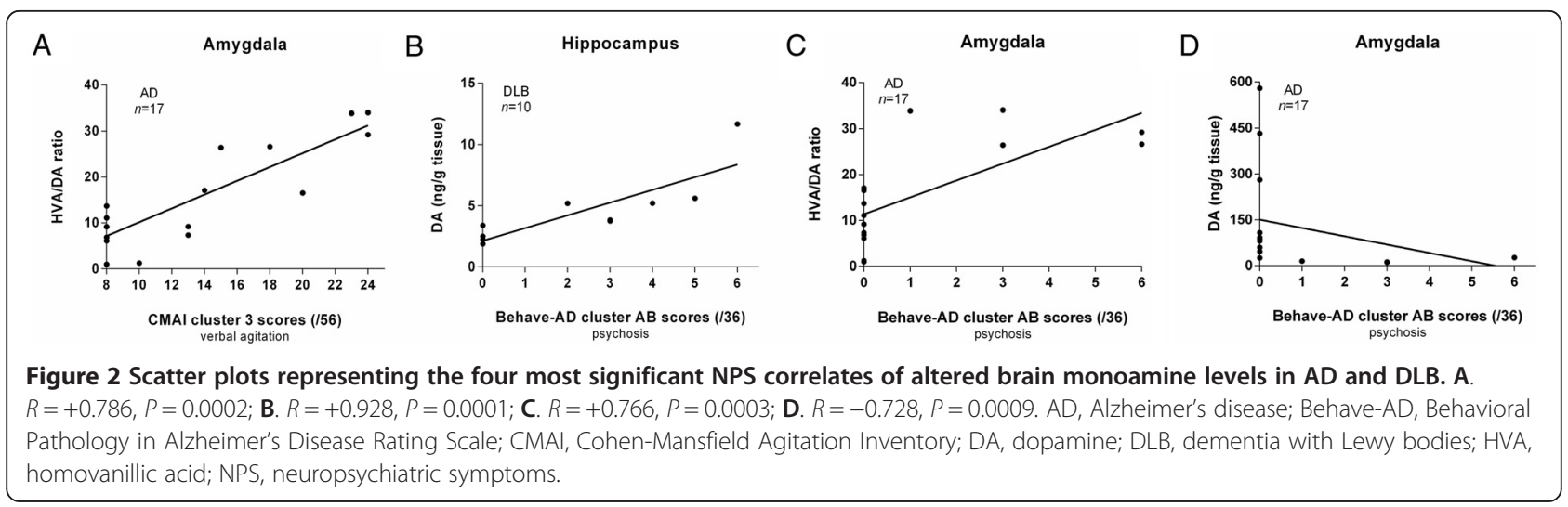




\section{Potential confounding monoaminergic effects of administered psychotropic medication}

In the $\mathrm{AD}+\mathrm{D}$ group, HVA levels of the amygdala (727.8 versus $537.0 \mathrm{ng} / \mathrm{ml} ; P=0.016)$, MHPG levels of BA17 (164.6 versus $119.6 \mathrm{ng} / \mathrm{ml} ; P=0.016)$ and MHPG (669.9 versus $531.4 \mathrm{ng} / \mathrm{ml} ; P=0.029)$ and HVA levels of the LC (1,690.0 versus $963.2 \mathrm{ng} / \mathrm{ml} ; P=0.029)$ were significantly higher in patients who were administered psychotropic medication before death $(n=4)$, compared to patients free of such medication $(n=5)$. In AD-D patients, only HVA/5-HIAA ratios of BA17 were revealed to be significantly lower in those patients who took psychotropic medication $(\mathrm{n}=7)$ compared to their medication-free counterparts $(\mathrm{n}=3)(0.259$ versus $0.541 ; P=0.017)$. With regard to the control subjects, only MHPG levels of BA22 were significantly increased in takers $(n=5)$ versus non-takers $(\mathrm{n}=5)(1,484.0$ versus $428.7 \mathrm{ng} / \mathrm{ml} ; P=0.032)$ and finally, in the DLB + D group, 5-HT levels of BA17 were significantly lower (2.443 versus $4.822 \mathrm{ng} / \mathrm{ml}$; $P=0.038$ ), whereas HVA levels of the cerebellar cortex (51.7 versus $20.8 \mathrm{ng} / \mathrm{ml} ; P=0.019$ ) as well as the HVA/ 5-HIAA ratios of BA9 (1.408 versus $0.459 ; P=0.038$ ), BA11 (0.839 versus $0.378 ; P=0.01)$ and BA24 $(0.685$ versus $0.325 ; P=0.01$ ) were all significantly higher in patients on psychotropic medication $(n=6)$ compared to patients free of such drugs $(n=4)$.

\section{Discussion}

\section{Study strengths and weaknesses}

Following our study protocol which allows no more than six hours between death and brain storage at $-80^{\circ} \mathrm{C}$, an average postmortem delay of approximately three to four hours was accomplished for each group. Besides this short time interval, additional $\mathrm{pH}$ measurements as a second quality control measure also ensured us of nonacidotic and high quality brain tissue samples. All groups were age- and gender-matched, with similar storage times of the frozen brain tissue samples and a low number of days between the last behavioral assessment and date of death (Table 1; see Results). Moreover, all the paraformaldehyde-fixated right brain hemispheres were always neuropathologically examined by the same neuropathologist and, in addition, regional brain dissections of the left frozen hemispheres were also carried out by the same scientist, which, respectively, minimized the diagnostic and inter-dissection variability. Our optimized RP-HPLC-ECD method was reliable, fast, robust and sensitive for the simultaneous determination of eight monoamines and metabolites with a low detection limit, high repeatability and recovery, short runtime, measurements in duplicate, short and simple sample preparation procedure and relatively high throughput. All analyses and sample preparation procedures were accurately performed by the same two scientists, which resulted in a low inter-assay variability.

With regard to the study weaknesses, each group consisted of a relatively small number of patients, although each patient was clinically, behaviorally and neuropathologically well characterized and nonparametric statistics were applied. Unfortunately, no behavioral ratings were available for the control subjects, although their clinical records did not demonstrate any evidence of psychiatric antecedents or depression. The absence of a nondepressed DLB group for neurochemical and statistical group comparisons might also be regarded as a study weakness. Furthermore, the administration of psychotropic medication before death might have camouflaged certain NPS and might also have altered the brain's monoamine levels and -receptors. Our neurochemical data thus might have been influenced, since the neurochemical effects may last up to several weeks after the last administration. In order to address this weakness, we applied a total Bonferroni correction to all statistical analyses and compared the brain monoamine levels between patients taking and not taking psychotropic medication in each group. Accordingly, these analyses showed that all of the highly significant aforementioned brain monoamine differences between the groups were most likely not influenced by psychotropic drugs, except HVA levels in the LC of AD + D patients and 5-HT and HVA levels in BA17 and the cerebellum, respectively, of $\mathrm{DLB}+\mathrm{D}$ patients. These specific monoamines were significantly altered in $\mathrm{AD}+\mathrm{D}$ and $\mathrm{DLB}+\mathrm{D}$ patients who were on psychotropic medication (see Results), which might have caused these same monoamines to become significantly altered in the $\mathrm{AD}+\mathrm{D} / \mathrm{DLB}+\mathrm{D}$ comparison (Table 2). Lastly, the concurrent AD pathology in eight of ten neuropathologically confirmed DLB patients might also form a limitation of this study, although the co-occurrence of $\mathrm{AD}$ and $\mathrm{DLB}$ is very common. More specifically, the neuropathological overlap between both neurodegenerative conditions is so extensive that 'pure' DLB, that is, without any AD-related pathology beyond that attributable to normal aging, is relatively uncommon, accounting for merely one third of all cases of Lewy body dementia and perhaps $4 \%$ of all dementia cases [64].

\section{The serotonergic neuropathophysiology of depression in DLB compared to AD}

Our results indicate that the serotonergic neurotransmission in the prefrontal, temporal, limbic and even occipital cortex as well as hippocampus might be severely impaired in DLB patients who suffered from depression. More specifically, we observed the lowest 5-HT and 5HIAA levels as well as 5-HIAA/5-HT turnover ratios in eight of eleven brain regions of DLB $+\mathrm{D}$ patients compared to $\mathrm{AD}+\mathrm{D}$ patients. 
In general, LB accumulate in the dorsal raphe nucleus, which is the largest serotonergic nucleus in the human brain and provides a substantial proportion of the serotonergic innervation to the forebrain [65], resulting in a marked reduction of 5-HT levels in the striatum, neocortex and frontal cortex of DLB patients [25]. Ballard and colleagues [3] also adduced that the pathophysiology of depression in DLB, as well as PDD, is fundamentally different from that seen in $\mathrm{AD}$, with alterations within the serotonergic pathway as a common feature of both. A previous study that investigated the theory of an impaired serotonergic neurotransmission in DLB patients with a major depressive disorder, observed an increased expression of 5-HT transporter (5-HTT) reuptake sites in BA7, the parietal neocortex, compared to those without depression [66]. On the other hand, in depressed AD patients, 5-HTT reuptake sites of the temporal cortex were reported to be significantly reduced compared to their nondepressed counterparts [67]. The depressed and nondepressed DLB groups of Ballard et al. [66] apparently also had lower mean 5-HTT reuptake site binding values than controls [unpublished observations; [25]]. The latter is comparable with our own serotonergic data, with significantly lower 5-HT and/or 5-HIAA levels in BA10, BA11, BA22, BA17 and cerebellum of $\mathrm{DLB}+\mathrm{D}$ patients compared to healthy controls. In other studies of Sharp et al., higher $5-\mathrm{HT}_{1 \mathrm{~A}}$ receptor binding densities were examined in postmortem brain tissue samples of the frontal cortex and BA36 in DLB patients compared to healthy controls (for the frontal cortex) [52] and in a subgroup of DLB + D subjects compared to DLB-D patients (for BA36) [28].

Based upon these recent findings, it seems plausible that in response to the severely damaged and degenerated raphe nuclei, a selective postsynaptic $5-\mathrm{HT}_{1 \mathrm{~A}}$ receptor upregulation and relative preservation of the 5-HTT reuptake sites might be triggered, specifically in depressed DLB patients, to compensate for the decreased serotonergic neurotransmission from these nuclei towards their neocortical and limbic projection areas $[3,25]$.

\section{Thalamic dopaminergic dysfunctioning and depression in DLB compared to AD}

Besides the serotonergic brain differences, our monoaminergic data also point to a decreased dopaminergic neurotransmission with four- and seven-fold increases of DOPAC/DA and HVA/DA turnover ratios and a ten-fold decrease of DA levels in the thalamus of DLB + D compared to $\mathrm{AD}+\mathrm{D}$ patients and control subjects. These findings in the thalamus might be attributed to the loss of DA producing neurons in the $\mathrm{SN}$, one of the neuropathological hallmarks in DLB, although a significant reduction of nigro-thalamic and -striatal DA levels may also mediate some of the non-motor symptoms [29], such as depression.
With regard to apathy, for example, David and colleagues [30] studied the relationship between apathy and striatal DA transporter (DAT) uptake in AD $(\mathrm{n}=14)$ and DLB $(\mathrm{n}=8)$ subjects by means of ${ }^{123}$ I-FP-CIT-SPECT imaging. The authors concluded that, using the Apathy Inventory, lack of initiative inversely correlated with bilateral putamen DAT uptake in the overall population of 22 patients. Although no statistical comparative analysis of the DAT binding potential (BP) values between DLB and AD was performed, their data clearly indicated that the subgroup of DLB subjects with apathy ( $n=6$; scores of 9 to 12) had much lower DAT BP values in left and right putamen than their apathetic AD counterparts ( $n=3$; scores of 9 to 12$)$. On the other hand, increased thalamic D2 receptor densities in 18 DLB patients compared to 14 elderly controls have been reported as well [29], although the authors did not determine thalamic DA levels nor classify their DLB population into depressed/nondepressed study patients.

Interestingly, Wilson et al. [68] also noticed a strong association between the LB density in the LC, dorsal raphe nucleus as well as $\mathrm{SN}$, and the degree of depressive symptoms in 124 older nondemented individuals. On the contrary, neurofibrillary tangle density within the same nuclei was not significantly related to depressive symptoms. Again, these results underline the prominent place of depression in synucleinopathies, such as DLB, and evidence the putative link between depression, LB and altered serotonergic and dopaminergic neurotransmitter systems.

\section{Psychosis and its dopaminergic pathogenesis in DLB versus $A D$}

Based upon our findings, we suggest that psychosis in AD might be pathophysiologically associated with a decreased dopaminergic neurotransmission solely restricted to the amygdala, whereas an impaired and rather increased dopaminergic activity across the mesolimbic system and LC might clinically account for psychosis in DLB.

The pathogenic link between altered dopaminergic pathways and psychosis in DLB has not been intensively investigated before. The most intriguing evidence comes from Roselli et al. [31], who observed an association between decreased striatal DAT levels, measured by ${ }^{123} \mathrm{I}-$ FP-CIT-SPECT imaging, and the presence of visual hallucinations in 18 DLB patients. When the authors considered the putamen and caudate nucleus separately, delusions, apathy and depression were inversely correlated with decreased caudate DAT levels also. The ratios of specific to nonspecific binding of ${ }^{123}$ I-FP-CIT, a DAT-specific radiotracer, were calculated as size-weighted averages of right, as well as left, brain activities. Similarly, our research group previously examined an inverse correlation between CSF HVA levels and hallucinations in a group of 26 DLB patients [33]. Overall, given the significantly impaired serotonergic neurotransmission in DLB brain [25], it is 
conceivable that the functional coupling between the serotonergic and dopaminergic pathways, with an inhibitory output of the serotonergic on the dopaminergic neurotransmission $[62,63]$, could be severely impaired, possibly leading to increased HVA and DA levels. Such a disruption of a functionally coupled system might explain the overall serotonergic and dopaminergic differences which are summarized in Table 3 . With regard to dopaminergic medications, to date, no randomized controlled trials have been performed to evaluate the use of levodopa to relieve psychosis in DLB, given the known side effects and the potential risk of worsening hallucinations and sleep disturbances [3]. For the same reasons, only a limited number of studies have investigated the use of antipsychotics in DLB patients, with one study reporting partial or complete amelioration of psychosis in $90 \%$ of participants after the administration of quetiapine [69], with only mild worsening in $27 \%$ of the cases of parkinsonian symptoms, while another one showed diminution of delusions and hallucinations after treatment with olanzapine [70].

Lastly, we need to bear in mind that the pathogenesis of psychosis in DLB is mainly related to right, and not left, hemispheric abnormalities, something which has been postulated after various SPECT and PET-related imaging studies $[53,71,72]$. Nonetheless, a left hemispheric hypoperfusion in the hippocampus, insula, ventral striatum and prefrontal, parietal and occipital cortices has also been previously linked with the presence of delusions and visual hallucinations in DLB [53].

\section{Brain MHPG levels are significantly reduced in DLB as opposed to $A D$}

DLB + D patients had significantly decreased brain MHPG levels compared to the $\mathrm{AD}+\mathrm{D}$ and/or $\mathrm{AD}-\mathrm{D}$ group(s) in almost eight of eleven brain regions. The most remarkable decrease was noticed in BA9 and BA10, BA22 and especially thalamus. Surprisingly, in BA17, MHPG levels were significantly increased in contrast with $\mathrm{AD}$ patients (Figure 1).

Reduced NA concentrations in the putamen, as well as frontal, temporal and occipital cortices of DLB patients compared to $\mathrm{AD}$ and control subjects have been previously observed by Ohara et al. [54], although no MHPG levels were measured and only five patients per group were included. Accordingly, MHPG and NA levels across the cortex of AD subjects have been reported to be significantly increased and decreased, respectively, compared to control values [55]. However, the only study that comes close in comparing brain MHPG levels between $\mathrm{DLB}$ and $\mathrm{AD}$ patients, is that from Langlais et al. [56], in which lower MHPG and NA levels in the caudate nucleus, putamen and BA8 (frontal cortex) of $\mathrm{LB}$ variant $\mathrm{AD}$ cases compared to 'pure' $\mathrm{AD}$ cases and control subjects were observed.
The importance of MHPG in DLB as opposed to AD has been emphasized before by Herbert et al. [73], who confirmed that the addition of MHPG to CSF amyloid beta $_{1-42}\left(A \beta_{1-42}\right)$, total- and phosphorylated tau protein improves the discrimination of DLB from AD but not from vascular dementia. These authors also measured CSF levels of MHPG, HVA and 5-HIAA and found that not only MHPG, but also HVA, as well as 5-HIAA were significantly lower in DLB compared to AD patients, which is very similar to our results (Table 2) [74]. Given the neuropathological hallmark of substantial LC neurodegeneration in DLB [75], our results underpin the hypothesis that MHPG might indeed have an important added biomarker value to effectively distinguish between both neurodegenerative conditions. Noteworthy, in our study, MHPG levels in the LC did not significantly differ, although NA levels and MHPG/NA ratios were significantly decreased and increased, respectively, in DLB compared to both AD subgroups. Results possibly demonstrate that the presence of $\mathrm{LB}$ in addition to plaques and tangles might further affect the noradrenergic neurotransmission in the LC. Moreover, in AD, compensatory noradrenergic changes in response to the significant noradrenergic cell body loss in the LC have been suggested [75]. Overall, both counteracting pathophysiological processes may have resulted in seemingly unaltered MHPG levels.

Finally, the surprisingly higher MHPG levels in BA17 of DLB versus AD patients may be indicative of certain distinct monoaminergic neurotransmitter alterations in the visual association cortex. Functional deficits of such a distinguishable cerebral network have been linked to the presence of psychosis in DLB before [53,76]. However, this assumption necessitates further investigation, certainly regarding its monoaminergic etiology.

\section{Conclusions}

By and large, we conclude that monoaminergic neurotransmitter alterations contribute differently to the neurochemical pathophysiology of depression and psychosis in DLB compared to AD. More specifically, our results support the hypothesis of a dysfunctional, decreased serotonergic neurotransmission as the main monoaminergic etiology of depression in DLB. The serotonergic pathways could potentially be even more impaired in DLB + D compared to those in $\mathrm{AD}+\mathrm{D}$, although the 5-HT deficiency theory may serve as a common feature of both. Moreover, given the generally decreased 5-HT and 5-HIAA levels, as well as 5-HIAA/5-HT turnover ratios, measured in our study, combined with the formerly suggested counteracting mechanisms of upregulated postsynaptic $5-\mathrm{HT}_{1 \mathrm{~A}}$ receptors and relatively preserved 5 -HTT reuptake sites, a concurrent pharmacological treatment using an SSRI and a $5-\mathrm{HT}_{1 \mathrm{~A}}$ receptor antagonist might indeed effectively 
alleviate depressive symptoms in DLB patients [25]. Correspondingly, we point at a severely impaired thalamic dopaminergic neurotransmission which, supplemented with a deficient nigrostriatal dopaminergic innervation, might mediate non-parkinsonian symptoms, such as depression, in DLB as well. Furthermore, we suggest that a generally increased dopaminergic neurotransmitter activity in the prefrontal, temporal and mesolimbic cortices as well as LC and hippocampus could closely relate to psychosis in DLB. On the contrary, in AD, a decreased dopaminergic neurotransmission and increased dopaminergic catabolism, specifically in the amgydala, might function as a monoaminergic substrate of psychosis. The complexity of an altered coupling between serotonergic and dopaminergic pathways might, additionally, also account differently for the presence of psychosis in DLB compared to that in $\mathrm{AD}$. On the whole, evidence is accumulating that future pharmacological treatments may target both serotonergic and dopaminergic systems to reduce these specific NPS. Finally, significantly lower MHPG levels across the brain, with the exception of BA17, in DLB compared to AD patients empathically stress its added CSF biomarker value to the traditional $A \beta_{1-42}$ and tau proteins, as was postulated before [73].

As for future studies, the functional importance of the basal ganglia and nigrostriatal pathways in non-motor symptoms in DLB necessitate further examination, given their monoaminergic relationship with depression, among others. For example, a neurochemical analysis of the entire neostriatum, subthalamic nucleus, $\mathrm{SN}$, thalamus, midbrain and cerebral cortex might create a better perspective on certain dysfunctional monoaminergic patterns which could contribute to NPS in DLB. With regard to psychosis in particular, inclusion of the right hemisphere is advised. Likewise, the relationship between NPS and acetylcholinergic neurotransmitter abnormalities in DLB as opposed to $\mathrm{AD}$ needs to be scrutinized as well.

\footnotetext{
Abbreviations

5-HIAA: 5-hydroxy-3-indoleacetic acid; 5-HT: 5-hydroxytryptamine (serotonin); 5-HTT: serotonin transporter; A: adrenaline; AD: Alzheimer's disease; AD + D/-D: AD patients with/without depression; $A D+D+P$ : psychotic AD patients with depression; AD-D-P: nonpsychotic AD patients without depression; $A \beta$ : amyloid beta; BA: Brodmann area; Behave-AD: Behavioral Pathology in Alzheimer's Disease Rating Scale; CMAI: Cohen-Mansfield Agitation Inventory; CSDD: Cornell Scale for Depression in Dementia; CSF: cerebrospinal fluid; DA: dopamine; DAT: dopamine transporter; DLB: Dementia with Lewy bodies; DLB + D/-D: DLB patients with/without depression; DLB + D + P: psychotic DLB patients with depression; DLB + D-P: nonpsychotic DLB patients with depression; DOPAC: 3,4dihydroxyphenylacetic acid; GDS: Global Deterioration Scale; HE: hematoxylin \& eosin; HVA: homovanillic acid; LB: Lewy bodies; LC: Iocus coeruleus; MFS: Middelheim Frontality Score; MHPG: 3-methoxy-4-hydroxyphenylglycol; MMSE: Mini-mental State Examination; NA: noradrenaline; NPS: neuropsychiatric symptoms; PDD: Parkinson's disease dementia; RP-HPLC-ECD: reversed-phase high-performance liquid chromatography with electrochemical detection; SN: substantia nigra; SSRI: selective serotonin reuptake inhibitor.
}

\section{Competing interests}

The authors declare that they have no competing interests.

\section{Authors' contributions}

Y , DVD, TA, SE, JJM and PPDD all made substantial contributions to the conception and design, and were involved in drafting the manuscript and/or revising it critically. The authors were also responsible for the acquisition, analysis and/or interpretation of data: $\mathrm{W}$ and SE behaviorally rated the dementia patients and assisted at performing the brain autopsies; PPDD and SE recruited the included patients and control subjects; PPDD, YV and SE were responsible for the acquisition and storage of samples; $W$ regionally dissected the left hemispheres; JJM meticulously performed all the neuropathological analyses and diagnoses; YV and TA completed all RP-HPLC-ECD analyses and calculations. W and DVD performed all the statistical analyses and interpreted the results. All authors read and approved the final manuscript, were responsible for the intellectual content, and agreed to be accountable for all aspects of the work.

\section{Acknowledgments}

This work was supported by a research grant of the Research FoundationFlanders (FWO), Interuniversity Poles of Attraction (IAP Network P7/16) of the Belgian Federal Science Policy Office, Methusalem excellence grant of the Flemish Government, agreement between Institute Born-Bunge and University of Antwerp, the Medical Research Foundation Antwerp, the Thomas Riellaerts research fund, and Neurosearch Antwerp. DVD is a postdoctoral fellow of the FWO. The authors acknowledge the contribution and support of all patients, relatives, caregivers, nursing personnel and clinical staff involved.

\section{Author details}

'Laboratory of Neurochemistry and Behavior, Institute Born-Bunge, University of Antwerp, Campus Drie Eiken, Universiteitsplein 1, Wilrijk, Antwerp 2610, Belgium. ${ }^{2}$ Department of Neurology and Memory Clinic, Hospital Network Antwerp (ZNA) Middelheim and Hoge Beuken, Lindendreef 1, Antwerp 2020, Belgium. ${ }^{3}$ Department of Neurology and Alzheimer Research Center, University of Groningen, University Medical Center Groningen (UMCG), Hanzeplein 1, Groningen 9713, GZ, The Netherlands. ${ }^{4}$ Biobank, Institute Born-Bunge, University of Antwerp, Universiteitsplein 1, Antwerp 2610, Belgium.

Received: 18 June 2014 Accepted: 16 December 2014

Published online: 11 February 2015

\section{References}

1. Vann Jones SA, O'Brien JT. The prevalence and incidence of dementia with Lewy bodies: a systematic review of population and clinical studies. Psychol Med. 2014;44:673-83.

2. McKeith IG, Burn DJ, Ballard CG, Collerton D, Jaros E, Morris CM, et al. Dementia with Lewy bodies. Semin Clin Neuropsychiatry. 2003;8:46-57.

3. Ballard C, Aarsland D, Francis P, Corbett A. Neuropsychiatric symptoms in patients with dementias associated with cortical Lewy bodies: pathophysiology, clinical features, and pharmacological management. Drugs Aging. 2013;30:603-11

4. McKeith IG, Dickson DW, Lowe J, Emre M, O'Brien JT, Feldman H, et al. Diagnosis and management of dementia with Lewy bodies: third report of the DLB Consortium. Neurology. 2005;65:1863-72.

5. Ballard C, Holmes C, McKeith I, Neill D, O'Brien J, Cairns N, et al. Psychiatric morbidity in dementia with Lewy bodies: a prospective clinical and neuropathological comparative study with Alzheimer's disease. Am J Psychiatry. 1999;156:1039-45.

6. Klatka LA, Louis ED, Schiffer RB. Psychiatric features in diffuse Lewy body disease: a clinicopathologic study using Alzheimer's disease and Parkinson's disease comparison groups. Neurology. 1996;47:1148-52.

7. Aarsland D, Brønnick K, Ehrt U, De Deyn PP, Tekin S, Emre M, et al. Neuropsychiatric symptoms in patients with Parkinson's disease and dementia: frequency, profile and associated caregiver stress. J Neuro Neurosurg Psychiatry. 2007;78:36-42.

8. Fritze F, Ehrt U, Hortobagyi I, Ballard C, Aarsland D. Depressive symptoms in Alzheimer's disease and Lewy body dementia: a one-year follow-up study. Dement Geriatr Cogn Disord. 2011;32:143-9.

9. Tun SM, Murman DL, Long HL, Colenda CC, von Eye A. Predictive validity of neuropsychiatric subgroups on nursing home placement and survival in patients with Alzheimer disease. Am J Geriatr Psychiatry. 2007;15:314-27.

10. Emanuel JE, Lopez OL, Houck PR, Becker JT, Weamer EA, Demichele-Sweet MA, et al. Trajectory of cognitive decline as a predictor of psychosis in early 
Alzheimer's disease in the cardiovascular health study. Am J Geriatr Psychiatry. 2011;19:160-8.

11. Russ TC, Batty GD, Starr JM. Cognitive and behavioural predictors of survival in Alzheimer disease: results from a sample of treated patients in a tertiaryreferral memory clinic. Int J Geriatr Psychiatry. 2012;27:844-53.

12. Mosimann UP, McKeith IG. Dementia with Lewy bodies - diagnosis and treatment. Swiss Med Wkly. 2003;133:131-42.

13. De Deyn PP, Drenth AF, Kremer BP, Oude Voshaar RC, Van Dam D. Aripiprazole in the treatment of Alzheimer's disease. Expert Opin Pharmacother. 2013;14:459-74.

14. Lyketsos CG, Carrillo MC, Ryan MJ, Khachaturian AS, Trzepacz P, Amatniek J, et al. Neuropsychiatric symptoms in Alzheimer's disease. Alzheimers Dement. 2011;7:532-9.

15. Rosenberg PB, Mielke MM, Han D, Leoutsakos JS, Lyketsos CG, Rabins PV, et al. The association of psychotropic medication use with the cognitive, functional, and neuropsychiatric trajectory of Alzheimer's disease. Int J Geriatr Psychiatry. 2012;27:1248-57.

16. Pollock BG, Mulsant BH, Rosen J, Sweet RA, Mazumdar S, Bharucha A, et al. Comparison of citalopram, perphenazine, and placebo for the acute treatment of psychosis and behavioral disturbances in hospitalized, demented patients. Am J Psych. 2002;159:460-5.

17. Pollock BG, Mulsant BH, Rosen J, Mazumdar S, Blakesley RE, Houck PR, et al. A double-blind comparison of citalopram and risperidone for the treatment of behavioral and psychotic symptoms associated with dementia. Am J Geriatr Psychiatry. 2007;15:942-52.

18. Finkel SI, Mintzer JE, Dysken M, Krishnan KR, Burt T, McRae T. A randomized, placebo-controlled study of the efficacy and safety of sertraline in the treatment of the behavioral manifestations of Alzheimer's disease in outpatients treated with donepezil. Int J Geriatr Psychiatry. 2004;19:9-18.

19. Teri L, Logsdon RG, Peskind E, Raskind M, Weiner MF, Tractenberg RE, et al. Treatment of agitation in AD: a randomized, placebo-controlled clinical trial. Neurology. 2000;55:1271-8.

20. Bhasin M, Rowan E, Edwards K, McKeith I. Cholinesterase inhibitors in dementia with Lewy bodies: a comparative analysis. Int J Geriatr Psychiatry. 2007;22:890-5

21. Grace J, Daniel S, Stevens T, Shankar KK, Walker Z, Byrne EJ, et al. Long-term use of rivastagmine in patients with dementia with Lewy bodies: an openlabel trial. Int Psychogeriatr. 2001;13:199-205.

22. McKeith I, Del Ser T, Spano P, Emre M, Wesnes K, Anand R, et al. Efficacy of rivastagmine in dementia with Lewy bodies: a randomized, double-blind, placebo-controlled international study. Lancet. 2000;356:2031-6.

23. Wild R, Pettit T, Burns A. Cholinesterase inhibitors for dementia with Lewy bodies. Cochrane Database Syst Rev. 2003;3:CD003672.

24. Rolinski M, Fox C, Maidment I, McShane R. Cholinesterase inhibitors for dementia with Lewy bodies: Parkinson's disease dementia and cognitive impairment in Parkinson's disease. Cochrane Database Syst Rev. 2012;3: CD006504.

25. Francis PT. Biochemical and pathological correlates of cognitive and behavioural change in DLB/PDD. J Neurol. 2009;256:280-5.

26. Ridha BH, Josephs KA, Rossor MN. Delusions and hallucinations in dementia with Lewy bodies: worsening with memantine. Neurology. 2005;65:481-2.

27. Sabbagh MN, Hake AM, Ahmed S, Farlow MR. The use of memantine in dementia with Lewy bodies. J Alzheimers Dis. 2005;7:285-9.

28. Sharp SI, Ballard CG, Ziabreva I, Piggott MA, Perry RH, Perry EK, et al. Cortical serotonin $1 \mathrm{~A}$ receptor levels are associated with depression in patients with dementia with Lewy bodies and Parkinson's disease dementia. Dement Geriatr Cogn Disord. 2008;26:330-8

29. Piggott MA, Ballard CG, Dickinson HO, McKeith IG, Perry RH, Perry EK. Thalamic D2 receptors in dementia with Lewy bodies, Parkinson's disease, and Parkinson's disease dementia. Int J Neuropsychopharmacol. 2007;10:231-44.

30. David R, Koulibaly M, Benoit M, Garcia R, Caci H, Darcourt J, et al. Striatal dopamine transporter levels correlate with apathy in neurodegenerative diseases - A SPECT study with partial volume effect correction. Clin Neurol Neurosurg. 2008;110:19-24

31. Roselli F, Pisciotta NM, Perneczky R, Pennelli M, Aniello MS, De Caro MF, et al. Severity of neuropsychiatric symptoms and dopamine transporter levels in dementia with Lewy bodies: a ${ }^{123}$ I-FP-CIT SPECT study. Mov Disord. 2009;24:2097-103.

32. Vermeiren $Y$, Van Dam D, Aerts T, Engelborghs S, De Deyn PP. Brain-region specific monoaminergic correlates of neuropsychiatric symptoms in Alzheimer's disease. J Alzheimers Dis. 2014;41:819-33.
33. Vermeiren $Y$, Le Bastard N, Van Hemelrijck A, Drinkenburg WH, Engelborghs S, De Deyn PP. Behavioral correlates of cerebrospinal fluid amino acid and biogenic amine neurotransmitter alterations in dementia. Alzheimers Dement. 2013:9:488-98.

34. Geda YE, Schneider LS, Gitlin LN, Miller DS, Smith GS, Bell J, et al. Neuropsychiatric symptoms in Alzheimer's disease: past progress and anticipation of the future. Alzheimers Dement. 2013;9:602-8.

35. Engelborghs S, Maertens K, Nagels G, Vloeberghs E, Mariën P, Symons A, et al. Neuropsychiatric symptoms of dementia: cross-sectional analysis from a prospective, longitudinal Belgian study. Int J Geriatr Psychiatry. 2005;20:1028-37.

36. McKhann GM, Drachman D, Folstein M, Katzman R, Price D, Stadlan EM. Clinical diagnosis of Alzheimer's disease: report of the NINCDS-ADRDA Work Group under the auspices of Department of Health and Human Services Task Force on Alzheimer's disease. Neurology. 1984;34:939-44.

37. McKhann GM, Knopman DS, Chertkow H, Hyman BT, Jack Jr CR, Kawas CH, et al. The diagnosis of dementia due to Alzheimer's disease: recommendations from the National Institute on Aging - Alzheimer's Association workgroups on diagnostic guidelines for Alzheimer's disease. Alzheimers Dement. 2011;7:263-9.

38. McKeith IG, Galasko D, Kosaka K, Perry EK, Dickson DW, Hansen LA, et al. Consensus guidelines for the clinical and pathologic diagnosis of dementia with Lewy bodies (DLB): report of the consortium on DLB international workshop. Neurology. 1996;47:1113-24.

39. American Psychiatric Association. DSM-IV-TR: Diagnostic and Statistical Manual of Mental Disorders. Washington DC: American Psychiatric Association; 2000.

40. Reisberg B, Borenstein J, Salob SP, Ferris SH, Franssen E, Georgotas A. Behavioral symptoms in Alzheimer's disease: phenomenology and treatment. J Clin Psychiatry. 1987;48:9-15.

41. De Deyn PP, Engelborghs S, Saerens J, Goeman J, Mariën P, Maertens K, et al. The Middelheim Frontality Score: a behavioural assessment scale that discriminates frontotemporal dementia from Alzheimer's disease. Int J Geriatr Psychiatry. 2005;20:70-9.

42. Cohen-Mansfield J, Marx MS, Rosenthal AS. A description of agitation in a nursing home. J Gerontol. 1989;44:77-84.

43. Alexopoulos GS, Abrams RC, Young RC, Shamoian CA. Cornell Scale for Depression in Dementia. Biol Psychiatry. 1988;23:271-84.

44. Reisberg B, Ferris SH, de Leon MJ, Crook T. The Global Deterioration Scale for assessment of primary degenerative dementia. Am J Psychiatry. 1982;139:1136-9.

45. Burns A, Lawlor BA, Craig S. Assessment scales in old age psychiatry. 2nd ed. London: Martin Dunitz; 2004.

46. Hollingworth P, Sweet R, Sims R, Harold D, Russo G, Abraham R, et al. Genome-wide association study of Alzheimer's disease with psychotic symptoms. Mol Psychiatry. 2012;17:1316-27.

47. Engelborghs S, Sleegers K, Cras P, Brouwers N, Serneels S, De Leenheir E, et al. No association of CSF biomarkers with APOEepsilon4, plaque and tangle burden in definite Alzheimer's disease. Brain. 2007;130:2320-6.

48. Braak H, Braak E. Neuropathological stageing of Alzheimer-related changes. Acta Neuropathol. 1991:82:239-59.

49. Braak H, Alafuzoff I, Arzberger T, Kretzschmar H, Del Tredici K. Staging of Alzheimer's disease-associated neurofibrillary pathology using paraffin sections and immunohistochemistry. Acta Neuropathol. 2006;112:389-404.

50. Jellinger KA, Bancher C. Neuropathology of Alzheimer's disease: a critical update. J Neural Transm Suppl. 1998:54:77-95.

51. Montine TJ, Phelps CH, Beach TG, Bigio EH, Cairns NJ, Dickson DW, et al. National Institute on Aging-Alzheimer's Association guidelines for the neuropathologic assessment of Alzheimer's disease: a practical approach. Acta Neuropathol. 2012;123:1-11.

52. Sharp SI, Ballard CG, Ziabreva I, Perry EK, Aarsland D, Larsen JP, et al. Serotonin 1-A receptor binding to frontal cortex in dementia with Lewy bodies and Parkinson's disease dementia. pA2 online Ejournal of the British Pharmacological. Society. 2005;2:65.

53. Nagahama Y, Okina T, Suzuki N, Matsuda M. Neural correlates of psychotic symptoms in dementia with Lewy bodies. Brain. 2010;133:557-67.

54. Ohara K, Kondo N, Ohara K. Changes of monoamines in post-mortem brains from patients with diffuse Lewy body disease. Prog Neuropsychopharmacol Biol Psychiatry. 1998;22:311-7.

55. Herregodts P, Bruyland M, De Keyser J, Solheid C, Michotte Y, Ebinger G. Monoaminergic neurotransmitters in Alzheimer's disease: an HPLC study comparing presenile familial and sporadic senile cases. J Neurol Sci. 1989:92:101-16. 
56. Langlais PJ, Thal L, Hansen L, Galasko D, Alford M, Masliah E. Neurotransmitters in basal ganglia and cortex of Alzheimer's disease with and without Lewy bodies. Neurology. 1993;43:1927-34.

57. Van Dam D, Vermeiren Y, Aerts T, De Deyn PP. Novel and sensitive reversed-phase high-pressure liquid chromatography method with electrochemical detection for the simultaneous and fast determination of eight biogenic amines and metabolites in human brain tissue. J Chromatogr A. 2014;1353:28-39.

58. Garcia-Alloza M, Tsang SW, Gil-Bea FJ, Francis PT, Lai MK, Marcos B, et al. Involvement of GABAergic system in depressive symptoms of Alzheimer's disease. Neurobiol Aging. 2006;27:1110-7.

59. Lewis DA. The human brain revisited: opportunities and challenges in postmortem studies of psychiatric disorders. Neuropsychopharmacology. 2002;26:143-54.

60. Monoranu CM, Apfelbacher M, Grünblatt E, Puppe B, Alafuzoff I, Ferrer I, et al. pH measurement as quality control on human post mortem brain tissue: a study of the BrainNet Europe consortium. Neuropathol Appl Neurobiol. 2009;35:329-37.

61. Stan AD, Ghose S, Gao XM, Roberts RC, Lewis-Amezcua K, Hatanpaa K, et al. Human postmortem tissue: what quality markers matter? Brain Res. 2006;1123:1-11.

62. Jenner $P$, Sheehy M, Marsden CD. Noradrenaline and 5-hydroxytryptamine modulation of brain dopamine function: implications for the treatment of Parkinson's disease. Br J Clin Pharmacol. 1983;15:277-89.

63. Kelland MD, Freeman AS, Chiodo LA. Serotonergic afferent regulation of the basic physiology and pharmacological responsiveness of nigrostriatal dopamine neurons. J Pharmacol Exp Ther. 1990;253:803-11.

64. Mrak RE, Griffin WS. Dementia with Lewy bodies: definition, diagnosis and pathogenic relationship to Alzheimer's disease. Neuropsychiatr Dis Treat. 2007;3:619-25

65. Hornung JP. The human raphe nuclei and the serotonergic system. J Chem Neuroanat. 2003;26:331-43.

66. Ballard C, Johnson M, Piggott M, Perry R, O'Brien J, Rowan E, et al. A positive association between $5 \mathrm{HT}$ re-uptake binding sites and depression in dementia with Lewy bodies. J Affect Disord. 2002;69:219-23.

67. Chen CP, Alder JT, Bowen DM, Esiri MM, McDonald B, Hope T, et al. Presynaptic serotonergic markers in community-acquired cases of Alzheimer's disease: correlations with depression and neuroleptic medication. J Neurochem. 1996;66:1592-8.

68. Wilson RS, Nag S, Boyle PA, Hizel LP, Yu L, Buchman AS, et al. Brainstem aminergic nuclei and late-life depressive symptoms. JAMA Psychiatry. 2013;70:1320-8.

69. Fernandez HH, Trieschmann ME, Burke MA, Friedman $\mathrm{JH}$. Quetiapine for psychosis in Parkinson's disease versus dementia with Lewy bodies. J Clin Psychiatry. 2002;63:513-5.

70. Cummings $\mathrm{J}$, Street J, Masterman D, Clark WS. Efficacy of olanzapine in the treatment of psychosis in dementia with Lewy bodies. Dementia Geriatr Cognit Disord. 2002;13:67-73.

71. Perneczky R, Drzezga A, Boecker H, Förstl H, Kurz A, Häussermann P. Cerebral metabolic dysfunction in patients with dementia with Lewy bodies and visual hallucinations. Dementia Geriatr Cognit Disord. 2008;25:531-8.

72. Perneczky R, Drzezga A, Boecker H, Wagenpfeil S, Förstl H, Kurz A, et al. Right prefrontal hypotmetabolism predicts delusions in dementia with Lewy bodies. Neurobiol Aging. 2009;30:1420-9.

73. Herbert MK, Aerts MB, Kuiperij HB, Claassen JA, Spies PE, Esselink RA, et al. Addition of MHPG to Alzheimer's disease biomarkers improves differentiation of dementia with Lewy bodies from Alzheimer's disease but not other dementias. Alzheimers Dement. 2014;10:448-55.

74. Aerts MB, Esselink RA, Claassen JA, Abdo WF, Bloem BR, Verbeek MM. CSF tau, $A \beta 42$, and MHPG differentiate dementia with Lewy bodies from Alzheimer's disease. J Alzheimers Dis. 2011;27:377-84.

75. Szot P, White SS, Greenup JL, Leverenz JB, Peskind ER, Raskind MA Compensatory changes in the noradrenergic nervous system in the locus ceruleus and hippocampus of postmortem subjects with Alzheimer's disease and dementia with Lewy bodies. J Neurosci. 2006;26:467-78.

76. Mori T, Ikeda M, Fukuhara R, Nestor PJ, Tanabe H. Correlation of visual hallucinations with occipital rCBF changes by donepezil in DLB. Neurology. 2006:66:935-7.

\section{Submit your next manuscript to BioMed Central and take full advantage of:}

- Convenient online submission

- Thorough peer review

- No space constraints or color figure charges

- Immediate publication on acceptance

- Inclusion in PubMed, CAS, Scopus and Google Scholar

- Research which is freely available for redistribution 